\title{
Crosstalk Between Pyroptosis and Apoptosis in Hepatitis C Virus-induced Cell Death
}

OPEN ACCESS

Edited by:

Pei-Hui Wang

Shandong University, China

Reviewed by:

Marcin Poreba,

Wrockaw University of Science and

Technology, Poland

Huang Huihuang,

Fifth Medical Center of the PLA

General Hospital, China

Zeng Qiong Zhen,

Jinan University, China

*Correspondence:

Rodney S. Russell

rodney.russel/@med.mun.ca

${ }^{t}$ These authors have contributed equally to this work and share first authorship

Specialty section: This article was submitted to Viral Immunology, a section of the journal

Frontiers in Immunology

Received: 01 October 2021 Accepted: 10 January 2022

Published: 14 February 2022

Citation:

Wallace HL, Wang L, Gardner CL, Corkum CP, Grant MD, Hirasawa K

and Russell RS (2022) Crosstalk Between Pyroptosis and Apoptosis in Hepatitis C Virus-Induced Cell Death.

Front. Immunol. 13:788138.

doi: 10.3389/fimmu.2022.788138
Hannah L. Wallace ${ }^{1 \dagger}$, Lingyan Wang ${ }^{1 \dagger}$, Cassandra L. Gardner ${ }^{1}$, Christopher P. Corkum ${ }^{2}$, Michael D. Grant ${ }^{1}$, Kensuke Hirasawa ${ }^{1}$ and Rodney S. Russell ${ }^{1 *}$

${ }^{1}$ Immunology and Infectious Diseases, Division of Biomedical Sciences, Faculty of Medicine, Memorial University, St. John's, NL, Canada, ${ }^{2}$ Confocal Imaging/Flow Cytometry Unit, Medical Laboratories, Faculty of Medicine, Memorial University, St. John's, NL, Canada

Extensive inflammation in the liver is known to contribute to the pathogenesis of hepatitis $\mathrm{C}$ virus (HCV) infection. Apoptosis has, for a long time, been known to act as a mechanism of hepatocyte death, but our previous research also identified inflammasome-mediated pyroptosis in infected and uninfected bystander cells as an additional mechanism of HCVinduced cytopathicity. The purpose of this study was to investigate the mechanism of HCV-induced cell death and to determine the timing and relative contributions of apoptosis and pyroptosis during HCV infection. In a model employing a cell cultureadapted strain of JFH-1 HCV and Huh-7.5 hepatocyte-like cells, we found that pyroptosis occurred earlier than did apoptosis during infection. CRISPR knockout of NLRP3 resulted in decreased caspase-1 activation, but not complete elimination, indicating multiple sensors are likely involved in HCV-induced pyroptosis. Knockout of gasdermin-D resulted in increased activation of apoptosis-related caspase-3, suggesting potential crosstalk between the two cell death pathways. An unexpected decrease in activated caspase- 1 levels was observed when caspase- 3 was knocked out, implying that caspase-3 may have a role in the initiation of pyroptosis, at least in the context of HCV infection. Lower viral titres in culture fluids and increased ratios of intracellular to extracellular levels of infectious virus were observed in knockout versus wild-type Huh7.5 cells, suggesting that HCV may induce programmed cell death in order to enhance virus release from infected cells. These results contribute to the understanding of HCV pathogenesis and add to the increasing volume of literature suggesting various programmed cell death pathways are not mutually exclusive.

Keywords: pyroptosis, inflammasome, NLRP3, hepatitis C virus, HCV, apoptosis, programmed cell death, pathogenesis 


\section{INTRODUCTION}

Multiple forms of programmed cell death (PCD) have been demonstrated in the context of virus infection and it is generally believed that viruses utilize these mechanisms to induce disease (1-4). However, some reports detail how PCD is employed as a host response to contain the virus [reviewed in reference (5)]. Both apoptosis and pyroptosis have been implicated in both contexts.

Apoptosis is a non-inflammatory form of cell death that is mediated by executioner caspase- 3 and can be initiated via one of two pathways. The intrinsic pathway begins intracellularly in response to changes in the intracellular environment, including, but not limited to, mitochondrial or DNA damage, endoplasmic reticulum stress, or reactive oxygen species, resulting in formation of the apoptosome. In contrast, the extrinsic pathway depends on initiation by ligand-binding of a death receptor on the cell surface, leading to cleavage of caspase-8. Both pathways ultimately result in activation of executioner caspase- 3 which leads to cell shrinkage, condensation of chromatin, nuclear fragmentation, and formation of apoptotic bodies that are cleared by circulating macrophages [both pathways extensively reviewed in reference (6)]. Apoptosis has been described as immunologically silent while still having a role in pathogenesis induced by some viruses $(7,8)$.

Pyroptosis is mediated by an inflammasome, a proteincomplex consisting of a sensor, such as NLRP3 [nucleotide oligomerisation domain, leucine-rich repeat, pyrin-domain containing protein 3; (9)] or AIM2 (absent in melanoma 2), an adaptor (ASC; apoptosis-associated speck-like protein containing a CARD [caspase recruitment domain]), and caspase- 1 as the effector enzyme (10). Once assembled into an inflammasome, activated caspase-1 cleaves gasdermin-D (GSDM-D) into its mature, pore-inducing form, which ultimately facilitates cell swelling and subsequent cell lysis (11). Pyroptosis, in contrast to apoptosis, is considered to be proinflammatory and immunogenic (6). Classically, pyroptosis was thought to function only as an innate immunity mechanism [reviewed in reference (12)], although recent findings suggest pyroptosis may also play a role in viral pathogenesis $(3,13,14)$.

Despite availability of highly effective curative drug therapies for treating HCV infection, as many as 71 million people worldwide remain chronically infected, with many of these people unaware of their infected status (15). Some infected individuals who undergo direct-acting antiviral (DAA) therapy still develop worsening liver disease, including hepatocellular carcinoma, despite prior elimination of the virus $(16,17)$. Plasma levels of many inflammatory cytokines decrease following DAA treatment, with notable exceptions being pyroptosis-associated interleukin-18 (IL-18) and interleukin-1 $\beta$ [IL-1 $\beta$; (18)]. It is important to understand how this virus induces liver disease as the burden of HCV on healthcare is predicted to increase in the coming decade (19).

It is well established that non-inflammatory, caspase-3mediated apoptosis occurs in the context of HCV infection both in vitro $(20,21)$ and in vivo $(22,23)$, and that this form of cell death contributes to liver pathology associated with chronic HCV infection (23). Our group has previously demonstrated that hepatocyte-like Huh-7.5 cells undergo both apoptosis and pyroptosis when infected with cell culture-adapted HCV (HCVcc). We demonstrated involvement of the NLRP3 inflammasome as indicated by a decrease in pyroptotic cell death induced by HCV when infection occurred in the presence of an NLRP3 inhibitor [MCC950; (24)]. To follow up on these findings, the current study aimed to identify the relative timing of these forms of programmed cell death during HCV infection (determining if pyroptosis and apoptosis occur sequentially or concurrently), to confirm the involvement of pyroptosisassociated proteins NLRP3 and GSDM-D and apoptosisassociated caspase-3, and to determine whether programmed cell death has a role in viral spread during HCV infection in vitro.

\section{MATERIALS AND METHODS}

\section{Cell Culture}

Huh-7.5 cells (gift from Apath, LLC) were maintained at $37^{\circ} \mathrm{C}$ with $5 \% \mathrm{CO}_{2}$ in complete medium (CM) containing Dulbecco's Modified Eagle Medium (DMEM; with high glucose [4.5 g/L] and pyruvate; ThermoFisher Scientific, 11995073), supplemented with $10 \%$ fetal bovine serum (FBS; heatinactivated, ThermoFisher Scientific, 10438034) and 1\% penicillin/streptomycin (Millipore Sigma, P4333-100ML).

\section{Virus Stocks}

A cell culture-adapted strain of HCV, known as JFH1 $1_{\mathrm{T}}(25,26)$, was used for this study. To generate virus stocks, $1 \times 10^{6}$ Huh-7.5 cells were seeded in 10-cm culture dishes. Approximately $24 \mathrm{~h}$ later, cells were inoculated at a multiplicity of infection (MOI) of 1 and incubated for $3 \mathrm{~h}$, after which inoculum was replaced with fresh CM. Culture fluids were harvested three days post-infection (p.i.) and virus titre was determined using a limiting dilution focus-forming assay described below.

\section{CRISPR-Cas9-Mediated Genome Editing}

Single guide RNA (sgRNA) sequences were designed to knock out NLRP3 (NLRP3_F 5'-GCGAAGCAGCACTCATGCGAG-3', NLRP3_R 5'-CTCGCATGAGTGCTGCTTCGC-3'), GSDM-D (GSDM-D_F 5'-GCAGCGAGTACACATTCATTG-3', GSDMD_R 5'-CAATGAATGTGTGTACTCGCTGC-3'), or caspase-3 (CASP-3_F_5'-GTGAGTTTTCAGTGTTCTCCA-3', CASP3 _R _ 5' - TGGAGA A C A C T GA A A A C T C A C - 3') . Oligonucleotide pairs were annealed and ligated into the BbsI site of a psPCas9-2A-GFP backbone using a rapid DNA ligation kit (Roche, 11635379001). Plasmids were transformed into Escherichia coli (DH5 $\alpha$ competent E. coli; ThermoFisher Scientific, 18258012) and cultures were incubated overnight at $37^{\circ} \mathrm{C}$ in $\mathrm{LB}$ medium, supplemented with $100 \mu \mathrm{g} / \mathrm{mL}$ ampicillin. Plasmids were isolated and transfected into Huh-7.5 cells using Lipofectamine 3000 (Thermo Fisher, L3000075). Transfected cells were selected by fluorescence-activated cell sorting based on GFP expression. Cells were seeded by limiting dilution and knockout clones were expanded. 


\section{Immunofluorescence Microscopy}

Cells were seeded at a density of $1 \times 10^{5}$ cells per well in 2-well chamber slides (Fisher Scientific, 12-565-16). The following day, cells were infected with $\mathrm{HCV}$ at an MOI $=1$ or left uninfected. Cells were left for one, two, three, or four days following infection for time course experiments or left for three or four days for nontime course experiments. Caspase- 1 was visualised using a FAMFLICA caspase-1 inhibitor kit (ImmunoChemistry Technologies, product number 98), referred to as a caspase-1 probe (27-31). On the designated day, CM was removed from cells and replaced with 30X FAM-FLICA caspase-1 probe in CM. Probe was incubated with cells for $45 \mathrm{~min}$ at $37^{\circ} \mathrm{C}$. Following incubation, an additional $400 \mu \mathrm{L}$ of $\mathrm{CM}$ was added and cells were incubated for one additional hour. Culture fluids were removed, replaced with a fresh $200 \mu \mathrm{L}$ of $\mathrm{CM}$, and the cells incubated for $5 \mathrm{~min}$. Cells were then washed in their respective wells with $300 \mu \mathrm{L}$ of apoptosis wash buffer (ImmunoChemistry Technologies, product number 98) for $20 \mathrm{~min}$ at room temperature in the dark. Following washing, cells were fixed and permeabilised by treating each well with $200 \mu \mathrm{L}$ of $100 \%$ acetone for $45 \mathrm{sec}$ at room temperature. After fixation, slides were washed for $1 \mathrm{~min}$ in phosphate-buffered saline (PBS; $\mathrm{pH}=7.4$ ). The same fixation protocol was used for all staining, independent of caspase-1 probe use. If the probe was not used, cells were fixed prior to staining. $\mathrm{HCV}$ core protein and cleaved caspase- 3 were detected using anti-HCV core (1:200 dilution in 5\% bovine serum albumin [BSA] in PBS; Anogen, product MO-I40015B) and anti-caspase-3 antibodies (1:200 dilution in 5\% BSA in PBS; BD Biosciences, 559565), respectively. When the anti-HCV core antibody was used alone, slides were incubated for $20 \mathrm{~min}$ at room temperature. If the anti-caspase- 3 antibody was used either alone or in conjunction with the anti-HCV core antibody, slides were incubated overnight at $4^{\circ} \mathrm{C}$. Following incubation with primary antibodies, slides were rinsed in PBS for 5 min prior to a 20 -min incubation with secondary antibodies. For regular flourescence microscopy, when either the anti-HCV core or the anti-caspase- 3 antibodies were used with the caspase- 1 probe, goat anti-mouse or goat anti-rabbit Alexa Fluor ${ }^{\circledR} 594$ (Invitrogen, A-11020 or A-11037), respectively, was used as the secondary antibody. If the anti-HCV core antibody was used without the caspase-1 probe, a goat anti-mouse Alexa Fluor ${ }^{\circledR} 488$ (Invitrogen, A-11029) secondary antibody was used. For confocal microscopy, when both the anti-caspase- 3 and antiHCV core antibodies were used together, goat anti-mouse Alexa Fluor $^{\circledR} 647$ (Invitrogen, A32728) was used for HCV core and goat anti-rabbit Alexa Fluor ${ }^{\circledR} 594$ (Invitrogen, A-11037) was used for caspase-3. For cells stained with specific antibodies for cleaved GSDM-D (Asp275, Cell Signaling Technology, 36425S, used at 1:100 dilution) and HCV core (same as above), cells were incubated with the primary antibodies in 5\% BSA in PBS overnight at $4^{\circ} \mathrm{C}$. The next day, cells were stained with goat anti-rabbit Alexa Fluor ${ }^{\circledR} 488$ (Thermofisher, A11008) and a goat anti-mouse Alexa Fluor ${ }^{\circledR} 594$ (Invitrogen, A-11020) antibodies to detect cleaved GSDM-D and HCV core protein, respectively. For cells stained for cleaved poly (ADP-ribose) polymerase (PARP; Asp214, Cell Signaling Technology, D64E10, used at
1:100 dilution) and caspase-3 (same as above), cells were also incubated overnight under the same conditions. The next day, cells were stained using goat anti-rabbit Alexa Fluor ${ }^{\circledR} 488$ (Thermofisher, A11008) and goat anti-mouse Alexa Fluor ${ }^{\circledR}$ 594 (Invitrogen, A-11020) secondary antibodies to visualize cleaved PARP and caspase-3, respectively. All secondary antibodies were used at 1:100 dilution in PBS. An additional 5min wash in PBS was performed following secondary antibody staining for all conditions. Slides were mounted using Vectashield Hard Set mounting medium containing DAPI (Vecta Shield Mounting Media, Vector Laboratories, MJS BioLynx Inc., VECTH1400 or Vecta Shield Vibrance Mounting Media, VECTH180010). Slides were viewed using either a Zeiss Axio Imager.M2 immunofluorescence microscope or an Olympus Fluoview FV1000 laser scanning microscope or a Zeiss LSM 900 with Airyscan microscope.

\section{Western Blotting}

Cells were seeded at a density of $1 \times 10^{6}$ in $10-\mathrm{cm}$ culture dishes. On the following day, cells were infected at an MOI $=1$ or 0.1 , or left uninfected. Cells were then incubated for the desired period of time (one, two, three, or four days p.i.). On the designated day, cells were lysed with RIPA buffer containing protease inhibitors and mixed with loading buffer. Culture fluids were collected and concentrated by centrifugation using Millipore filters (Millipore, Amicon Ultra4 centrifugal filter units, Ultracel-10K, UFC801096). Prepared samples were heated at $100^{\circ} \mathrm{C}$ in a heating block for $5 \mathrm{~min}$. Proteins were separated by SDS-PAGE and transferred to nitrocellulose membranes (Amersham/Cytiva, 10600065). Membranes were blocked with $5 \%$ skimmed milk powder in TBST (1X TBS with $0.05 \%$ Tween-20) for $1 \mathrm{~h}$ at room temperature with agitation. Membranes were then incubated with antibodies recognising pro-caspase-3 (Santa Cruz, sc7272), cleaved caspase-3 (Cell Signaling Technology, 9664), pro-caspase1 (Santa Cruz, sc56036), cleaved caspase-1 (AdipoGen, AG-20B0048), HCV core protein (Anogen, MO-I40015B or C7-50, Thermofisher, MA1-080), GSDM-D (Santa Cruz, sc81868), cleaved PARP (Asp214, Cell Signaling Technology, D64E10), or GAPDH (Abcam, ab8245) overnight at $4^{\circ} \mathrm{C}$ at a 1:1000 dilution in TBST. On the following day, membranes were washed in TBST. HRP-conjugated anti-mouse (Santa Cruz, sc516102) or anti-rabbit (Santa Cruz, sc2357) secondary antibodies were incubated with the membranes at room temperature at a 1:5000 dilution in TBST for $1 \mathrm{~h}$. Signal was detected using ECL Western blotting substrates (Clarity Western ECL substrate, Bio-Rad, 170-5060; Amersham ECL select substrate GE Healthcare, RPN2235). All Western blot figures, with the exception of Figure 1F, were prepared from images obtained using ImageQuant LAS 4000 (GE Healthcare). The images in Figure 1F were obtained using Bio-Rad ChemiDoc Imaging System and processed using Image Lab (version 6.1.0, BioRad Laboratories, Inc.).

\section{Flow Cytometry}

Cells were seeded at a density of $1.5 \times 10^{5}$ in 6 -well plates. The following day, cells were infected with $\mathrm{HCV}$ at an MOI $=1$ or left uninfected. For a positive control, the day prior to staining, cells 
A

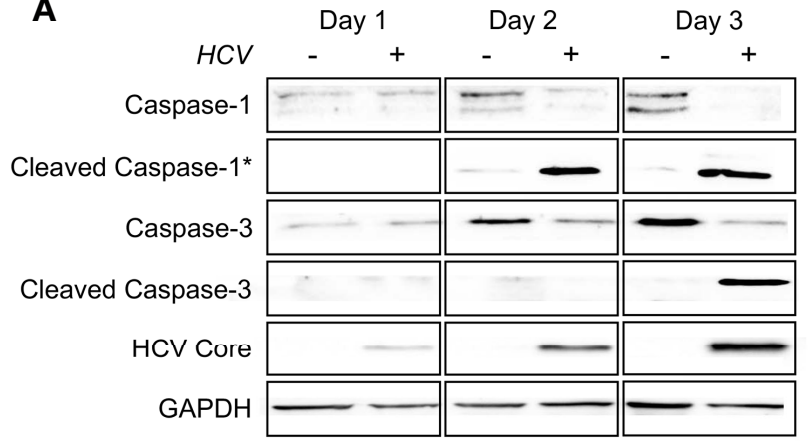

B
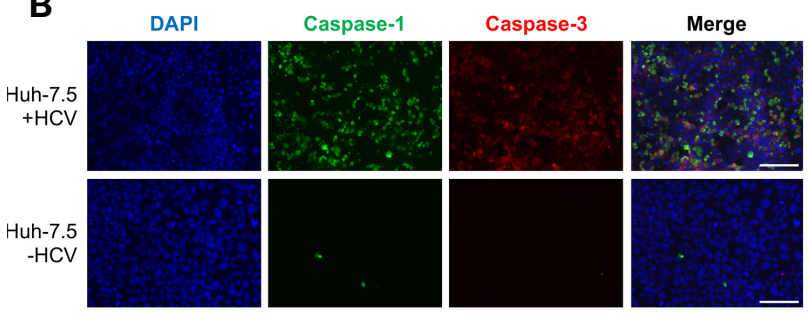

C

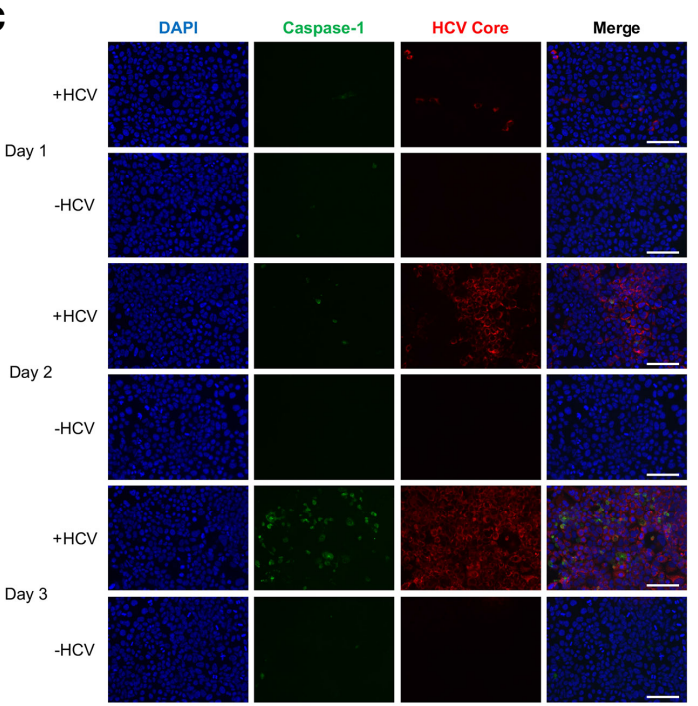

D

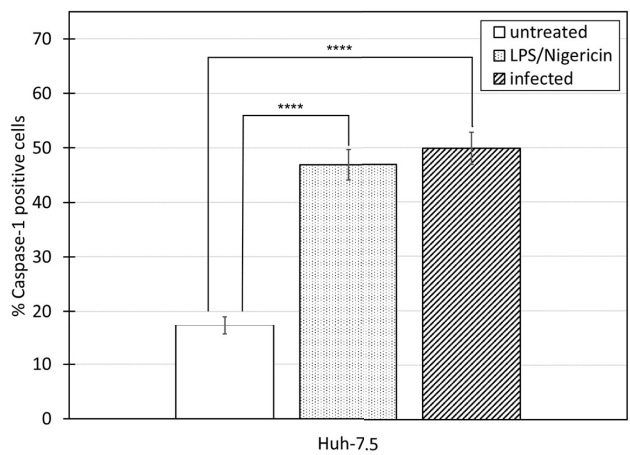

E

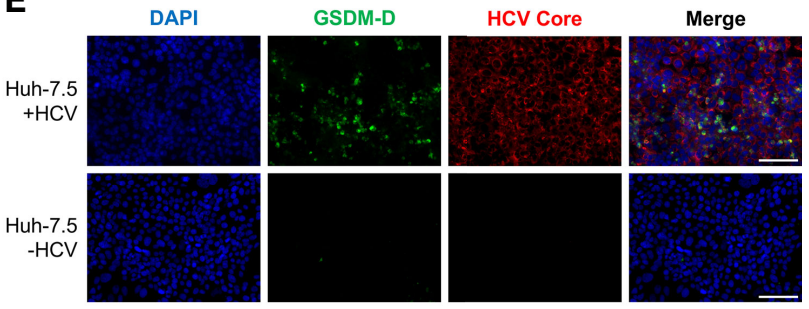

$\mathbf{F}$

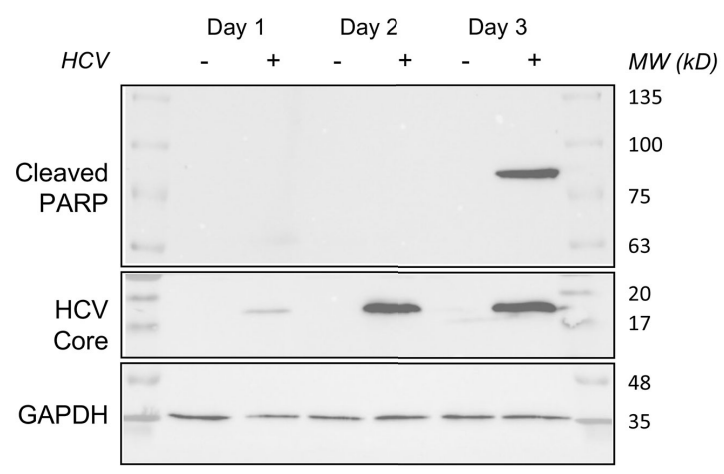

G
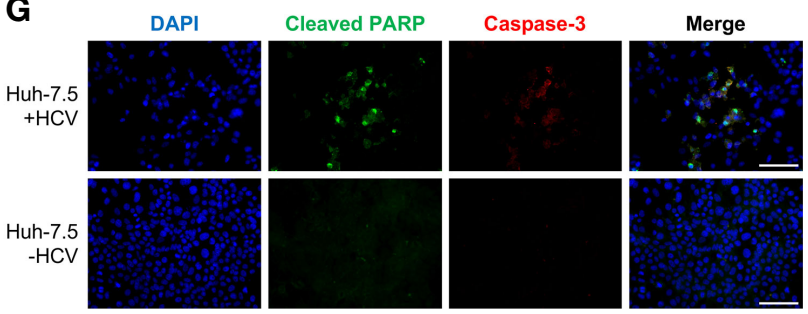

FIGURE 1 | Activation of pyroptosis- and apoptosis-associated proteins during HCV infection. Huh-7.5 cells were infected with HCV at MOI = 1 or left uninfected. (A) At 1, 2, and 3 days p.i., cells and culture fluids were harvested for Western Blot analysis. Membranes were probed for pro-caspase-1, cleaved-caspase-1, procaspase-3, cleaved-caspase-3, HCV core protein, and GAPDH. * indicates samples from cell culture fluids rather than cell lysates. (B) At 3 days p.i., cells were stained for cleaved caspase-1 (green) using a specific probe, then fixed using acetone. Cells were subsequently stained using antibodies specific for cleaved caspase-3 (red). Scale bar, $100 \mu \mathrm{m}$ (C) At 1, 2, and 3 days p.i., cells were stained for cleaved caspase-1 using the same specific probe (green) then fixed using acetone. Cells were subsequently stained using an antibody specific for HCV core protein (red). (B, C) Nuclei were stained with DAPI (blue) and analysis was performed using fluorescent microscopy. Scale bar, $100 \mu \mathrm{m}$ (D) At day 4 p.i., cells were stained for cleaved caspase-1 using a specific probe and fixed using fixative from the caspase-1 probe kit. Cells were run on a CytoFLEX flow cytometer and data were analyzed using Kaluza analysis software. Data are presented as the percent of total cells that were positive for caspase-1. ${ }^{\star \star \star \star} p<0.0001$. (E) At 3 days p.i., cells were fixed using acetone and stained for cleaved GSDM-D (green) and HCV core protein (red) using specific antibodies. Scale bar, $100 \mu \mathrm{m}$ (F) At 1, 2, and 3 days p.i., cells were harvested for Western Blot analysis. Membranes were probed for cleaved PARP, HCV core protein, and GAPDH. (G) At 3 days p.i., cells were fixed using acetone and stained for cleaved PARP (green) and cleaved caspase-3 (red) using specific antibodies. Scale bar, $100 \mu \mathrm{m}$ (A-G) Data are representative of three independent experiments. 
were treated with lipopolysaccharide from E. coli 0127:B8 used at 5 $\mu \mathrm{g} / \mathrm{mL}$ (LPS; in vitro LPS, Millipore-Sigma, L5024-10MG) for $3 \mathrm{~h}$, followed by addition of Nigericin used at $167.5 \mu \mathrm{M}$ (sodium salt, InvivoGen, tlrl-nig) and cultured overnight. At day four p.i., cell culture fluids were collected and cells trypsinised. Cells and culture fluids were centrifuged together at $400 \mathrm{x} g$ for $5 \mathrm{~min}$. Supernatant was discarded and cell pellets were resuspended in $200 \mu \mathrm{L}$ of $30 \mathrm{X}$ caspase-1 probe in CM (same FAM-FLICA caspase-1 inhibitor kit as above) and incubated for $45 \mathrm{~min}$ at $37^{\circ} \mathrm{C}$. Following incubation, an additional $400 \mu \mathrm{L}$ of $\mathrm{CM}$ was added and cells were incubated for one additional hour. Cells were then centrifuged at $400 \mathrm{x} g$ for $5 \mathrm{~min}$ and supernatants discarded. Cell pellets were then resuspended in $300 \mu \mathrm{L}$ of apoptosis wash buffer and cells were fixed by adding $60 \mu \mathrm{L}$ of fixative from the caspase-1 probe kit (ImmunoChemistry Technologies, product number 98). The cells were then incubated at room temperature in the dark for $20 \mathrm{~min}$. Following incubation, cells were centrifuged as above and supernatant was discarded. Cells were resuspended in $500 \mu \mathrm{L}$ PBS and stored at $4^{\circ} \mathrm{C}$ until analysis. Cells were run on a CytoFLEX flow cytometer (Beckman Coulter) and FAM-FLICA fluorescence was detected using the 525/40 detector on the $488 \mathrm{~nm}$ laser. Flow cytometry experiments performed on knockout cell lines were carried out concurrently and presented in different figures for analysis purposes. The percentage of cells that were positive for cleaved caspase- 1 in both the infected and uninfected wild-type Huh-7.5 cells were used for comparison purposes in each of the figures that includes flow cytometry data from the knockout cells. Analysis of flow cytometry data was done using Kaluza software (version 2.1.1; Beckman Coulter). Gating strategy and analysis protocol are detailed below.

\section{Virus Titre}

Extracellular virus titre was determined by performing a 10 -fold serial dilution of the virus stock, followed by infection in triplicate of Huh-7.5 cells in 8-well chamber slides that had been plated at a density of $5 \times 10^{4}$ cells per well on the previous day. After infection, cells were incubated for two days and then fixed using acetone. Slides were stained for HCV core protein (Anogen, MO-I40015B) using the same dilutions as above and incubated at room temperature for $20 \mathrm{~min}$. This was followed by incubation with goat anti-mouse Alexa Fluor ${ }^{\circledR} 488$, as described above. Slides were mounted with Vectashield containing DAPI. Virus titre was determined based on the number of foci present in the highest positive dilution and titre was then expressed as focus-forming units per millilitre (FFU/mL).

To measure intracellular infectious titre, both control and infected cells were harvested following a three-day infection at $\mathrm{MOI}=0.1$, pelleted by centrifugation for $5 \mathrm{~min}$ at $400 \times g$ and resuspended in $1 \mathrm{~mL}$ of $\mathrm{CM}$. The re-suspended cells were then lysed by three cycles of freeze/thaw ( 3 min freeze, dry ice/ methanol bath; $3 \mathrm{~min}$ thaw, $37^{\circ} \mathrm{C}$ water bath) and pelleted by centrifugation for $10 \mathrm{~min}$ at $1500 \times g$. Virus titres were determined as described above based on clarified supernatants.

\section{Flow Cytometry Analysis}

Controls employed to guide the analysis of flow cytometry data included unstained cells for each condition, LPS/Nigericin as a pyroptosis positive control, and heat shocked cells $\left(65^{\circ} \mathrm{C}\right.$ for $6 \mathrm{~min}$, sufficient to kill the majority of cells) as a positive control for cell death. Uninfected, unstained wild-type Huh-7.5 cells acted as the negative control. Wild-type Huh-7.5 cells were always analyzed in parallel when performing this assay on knockout cells and comparisons were all made by comparing wild-type to knockout cells.

The overall cell population was gated on the forward vs. side-scatter plot using an inclusive gate as Huh-7.5 cells are heterogeneous and would be expected to vary in size even in the absence of $\mathrm{HCV}$ infection. Gates for subpopulations of cells were generated on forward vs. side-scatter plots using heat shock controls and uninfected cell controls and applied to LPS/ Nigericin-treated controls. Two populations of cells were observed in our LPS/Nigericin treated control and these corresponded with the heat shocked cell population and the uninfected cell population. One population of cells was smaller (shifted down and to the left on the forward vs. side-scatter plot) and corresponded with the dead, heat-shocked cell sample. There was also a population of larger cells that had the same range of forward vs. side-scatter as the uninfected Huh-7.5 cells. As LPS/Nigericin stimulation is an established pyroptosis positive control, this two-population gating strategy guided further analysis of $\mathrm{HCV}$-infected cell samples. Within the HCV-infected cell samples, there were the same two distinct cell populations observed in the LPS/Nigericin-treated control. The majority of the caspase- 1 positive cells were located within the "small" population. However, it is important to note that not all the caspase- 1 positive cells were in this population, and percent caspase-1 positivity and mean fluorescence intensity varied between conditions. In order to have sufficient numbers of caspase- 1 positive cells that were representative of the whole sample, we recorded 10,000 events in the "small" population meaning at least 10,000 events were collected for each condition.

As dying cells take up the caspase- 1 probe non-specifically, the heat shock population was used to set the caspase-1 fluorescence positivity threshold. Uninfected, unstained Huh7.5 cells were used to gate out background fluorescence. These two gates were applied to the LPS/Nigericin-treated sample to ensure the gates were representative of caspase- 1 positivity. The same gates were applied to all conditions for analysis. For clarification purposes, images of the gating strategy are also included in the supplementary materials (Figure S1).

\section{Statistical Analyses}

Statistical analyses for flow cytometry data were performed using the Analysis ToolPak in Microsoft Excel (2016). Oneway ANOVA was used to compare conditions. Where p-values of $<0.05$ were considered statistically significant. All flow cytometry statistical analysis was evaluated using at least three independent experiments.

Statistical analyses for viral titre data were performed using SPSS statistics 27. One-way ANOVA with Bonferroni multiple comparison test was used to compare between multiple groups. p-values $<0.05$ were considered statistically significant. 


\section{RESULTS}

\section{HCV Infection Induces Both Pyroptosis and Apoptosis, With Pyroptosis Preceding Apoptosis}

Our previous research suggested that both apoptosis and pyroptosis were induced during HCV infection in vitro (24). To confirm this result and to elucidate the relative timing by which apoptosis and pyroptosis occur during HCV infection, infected $(\mathrm{MOI}=1)$ and uninfected cells, and culture fluids were compared for the presence of cleaved caspase-1, indicative of pyroptosis, and cleaved caspase-3, indicative of apoptosis, using Western blot analysis. This was accomplished by performing time-course experiments to compare cleaved caspase- 1 and -3 expression over time. We first established that HCV core protein levels increased with subsequent days p.i. (Figure 1A) which was confirmed using fluorescent microscopy (Figure 1C). Cleaved caspase-1 peaked at day two p.i. and remained consistent at day three p.i. in culture fluids taken from HCVinfected cell cultures (Figure 1A). This coincided with an expected decrease of caspase-1 (full-length) levels in HCVinfected cells at days two and three p.i. (Figure 1A). Levels of cleaved caspase- 1 were also shown to increase with increasing time p.i. in cells that remained adherent (Figure 1C). Apoptosis was initiated on day three p.i., indicated by a decrease in the level of caspase- 3 in HCV-infected cells at days two and three p.i., while cleaved caspase- 3 increased at day three p.i. (Figure 1A). Decreased expression of both caspase-1 and -3 coincided with an increase in their active, cleaved forms. These results were confirmed by fluorescence microscopy, using an antibody specific for cleaved caspase- 3 and a probe specific for cleaved caspase-1, demonstrating increased caspase- 1 and -3 activation in HCV-infected cells at three days p.i. when compared to uninfected controls (Figure 1B). Using flow cytometry to quantify the percentage of caspase-1-positive cells, we confirmed a significant difference in the percentage of caspase-1-positive cells between uninfected Huh-7.5 cells $(\sim 18 \%)$ and both HCV-infected ( $50 \%)$ and LPS/Nigericintreated ( 45\%) Huh-7.5 cells (Figures 1D, S2). To validate the use of caspase- 1 and -3 as markers of pyroptosis and apoptosis, respectively, we also verified caspase-1 cleavage of downstream GSDM-D and caspase- 3 cleavage of PARP. Cleavage of GSDMD was detected using an antibody specific for cleaved GSDM-D, which was observed at a greater level in infected cells than uninfected controls (Figure 1E). We attempted to show this by Western blot analysis but we were unsuccessful with our current antibody. In accordance with our results showing the activation of caspase-3, cleaved PARP was undetectable by Western blotting, even with extended exposure, until day 3 p.i. (Figure 1F). This was also confirmed using fluorescence microscopy with an antibody specific for cleaved PARP which showed increased PARP cleavage in HCV-infected cells when compared to uninfected controls (Figure 1G). Overall, these results confirm involvement of both apoptosis and pyroptosis during HCV infection and suggest that pyroptosis precedes apoptosis during infection progression.

\section{NLRP3 Is One of the Sensors Involved in HCV-Induced Pyroptosis}

Our previous work suggested that HCV-induced pyroptosis is mediated by the NLRP3 inflammasome since cell death was reduced when cells were treated with NLRP3 inhibitor, MCC950 (24). To further corroborate the involvement of NLRP3 in HCVmediated pyroptosis, the CRISPR-Cas9 system was used to knock out NLRP3 in Huh-7.5 cells (Figure S3). Both wild-type Huh-7.5 and NLRP3 knockout $(\mathrm{KO})$ cells were infected with HCV and Western blot analysis was performed on cells and cell culture fluids at one, two, and three days p.i. We observed decreased levels of caspase-1 on days two and three p.i. in infected NLRP3 KO cells compared to wild-type control cells. However, knockout of NLRP3 did not eliminate caspase-1 activation entirely (Figure 2A). Detection of cleaved caspase- 1 in cell culture fluids was delayed until day three p.i. in culture fluids from NLRP3 KO cells compared to wild-type controls. Similar results were confirmed by fluorescence microscopy, with the infected NLRP3 KO cells displaying diminished caspase-1 activation compared to wildtype Huh-7.5 cells at day three p.i. (Figures 2B, S4A). Diminished cleaved caspase-1 expression was even more prominent when the Western blotting was performed on samples from an infection performed at an MOI $=0.1$, likely due to the fact that activation of caspases is dependent on the extent of virus replication (Figure 2C). Western blot analysis and fluorescence microscopy revealed similar levels of cleaved caspase-3 at day three p.i. in both Huh-7.5 and NLRP3 KO cell lines when an MOI $=1$ was used (Figures 2A, B) but, when an MOI $=0.1$ was used, NLRP3 KO cells showed a reduction in levels of cleaved caspase-3 compared with wild-type cells at four days p.i. (Figure 2C). The difference in caspase-1 activation between Huh7.5 cells and NLRP3 KO cells was clear at day three p.i. using Western Blot analysis and fluorescence microscopy. However, using flow cytometry, we showed no significant difference in the percentage of caspase-1 positive cells between $\mathrm{HCV}$-infected Huh7.5 and NLRP3 KO cells at day four p.i. (Figures 2D, S4B). When fluorescence microscopy was performed on cells four days p.i., there was also no detectable difference in caspase- 1 activation between Huh-7.5 cells and NLRP3 KO cells (Figure 2E). Taken together, these results confirm involvement of NLRP3 in HCVinduced pyroptosis. The fact that caspase- 1 activation was not completely eliminated at day three p.i. and was detected at similar levels to that of wild-type Huh-7.5 cells on day four p.i., indicates that other sensors upstream of caspase-1 may also be involved in HCV-induced pyroptosis or the NLRP3 KO was incomplete.

\section{Apoptosis Increases in the Absence of Pyroptosis}

GSDM-D is a common component of the pyroptosis pathway across all sensors and is responsible for the final pore formation step leading to cell lysis (32-35). Early in our investigation, we raised the question as to what the outcome would be if one form of cell death was inhibited. Would the inhibition of pyroptosis cause cells to switch towards a different form of cell death, potentially indicating crosstalk between the two pathways (36-40)? To investigate the role of GSDM-D in HCV-induced pyroptosis and 
A

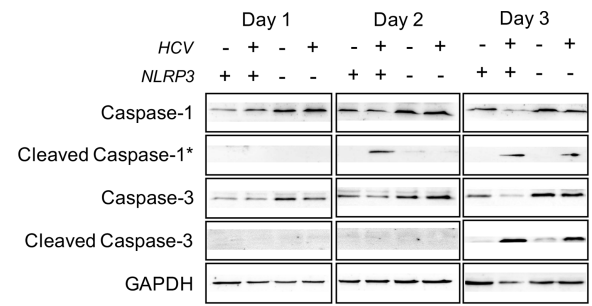

B

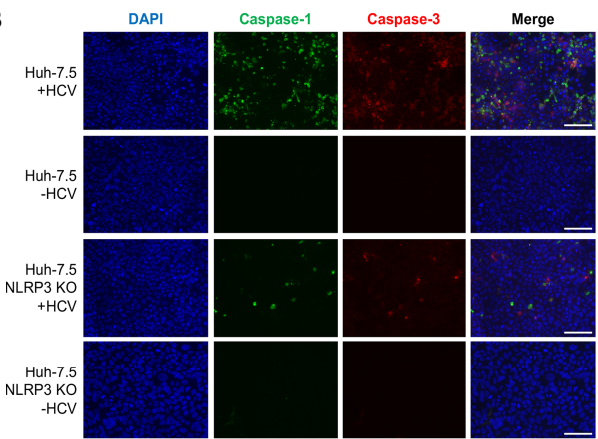

C

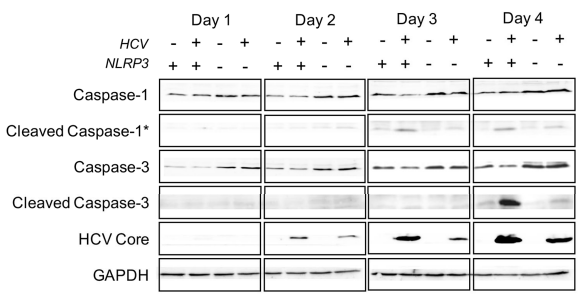

D

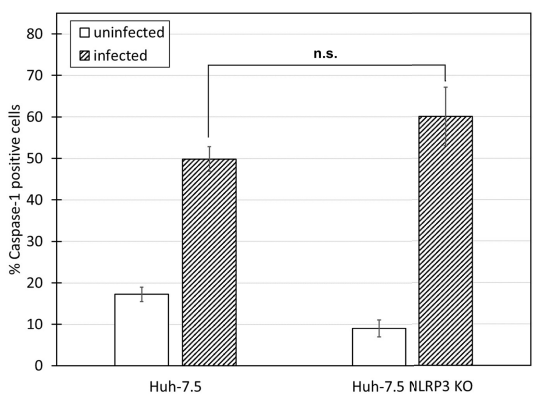

E

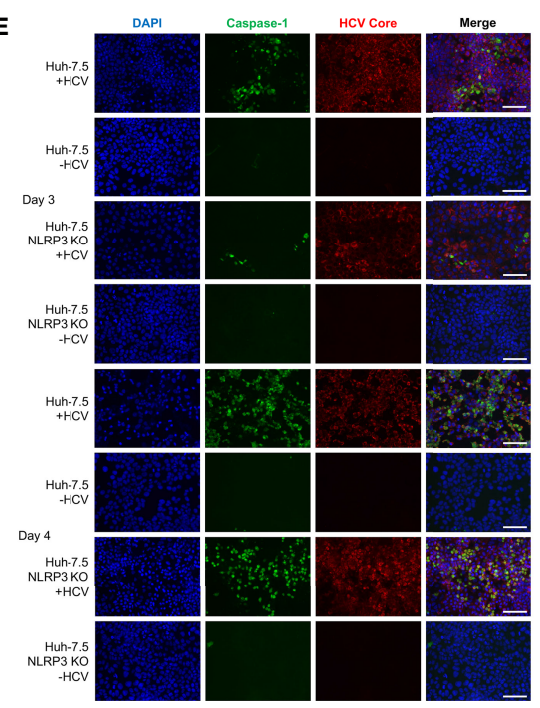

FIGURE 2 | Caspase-1 and -3 activation in Huh-7.5 cells and Huh-7.5 NLRP3 KO cells. (A, B) Huh-7.5 cells or Huh-7.5 NLRP3 KO cells were infected with HCV at $\mathrm{MOI}=1$ or left uninfected. (A) At 1, 2, and 3 days p.i., cells and culture fluids were harvested for Western Blot analysis. Membranes were probed for pro-caspase-1, cleaved-caspase-1, pro-caspase-3, cleaved-caspase-3, and GAPDH. * indicates samples from cell culture fluids rather than cell lysates. (B) At 3 days p.i., cells were stained for cleaved caspase-1 (green) using a specific probe then fixed using acetone. Cells were subsequently stained using an antibody specific for cleaved caspase-3 (red). Nuclei were stained with DAPI (blue) for analysis by fluorescence microscopy. Scale bar, 100 m (C) Huh-7.5 cells or Huh-7.5 NLRP3 KO cells were infected with $\mathrm{HCV}$ at $\mathrm{MOI}=0.1$ or left uninfected. At 1, 2, 3, and 4 days p.i., cells and culture fluids were harvested for Western Blot analysis. Membranes were probed for pro-caspase-1, cleaved-caspase-1, pro-caspase-3, cleaved-caspase-3, HCV core protein, and GAPDH. * indicates samples from cell culture fluids rather than cell lysates. (D) At day 4 p.i., cells were stained for cleaved caspase- 1 using a specific probe and fixed using fixative from the caspase- 1 probe kit. Cells were run on a CytoFLEX flow cytometer and data were analyzed using Kaluza analysis software. Data from NLRP3 KO cells are compared here to data from wild-type cells found in Figure 1D. Data are presented as the percent of total cells positive for caspase-1. $p=$ n.s. (E) At 3 and 4 days p.i., cells were stained for cleaved caspase-1 (green) using a specific probe, then fixed using acetone. Cells were subsequently stained using an antibody specific for HCV core protein (red). Nuclei were stained with DAPI (blue) for analysis by fluorescence microscopy. Scale bar, $100 \mu \mathrm{m}$ (A-E) Data are representative of three independent experiments.

to investigate the likelihood of crosstalk between the pyroptotic and apoptotic pathways, CRISPR-Cas9 was utilised to generate Huh7.5 cells lacking GSDM-D (Figure 3A). Wild-type and GSDM-D $\mathrm{KO}$ cells were infected with $\mathrm{HCV}$ at an MOI $=1$ (Figure 3A). Cells and culture fluids were harvested from infected and uninfected conditions at one, two, and three days p.i.. Western blot analysis of GSDM-D KO cell lysates revealed that levels of both caspase- 3 and cleaved caspase-3, indicative of apoptosis, increased at three days p.i. when compared to wild-type cells (Figure 3A). While the same increase of cleaved caspase- 3 was not found when fluorescence microscopy was performed, there was a change in the caspase- 3 staining morphology with more diffuse staining patterns (Figures 3B, S5A). These results suggest a change of cell death patterns induced by infection with HCV when GSDM-D has been knocked out whereby inhibition of one cell death pathway stimulated another. Results using fluorescence microscopy at three days p.i. and flow cytometry at four days p.i. both revealed non-significant differences in cleaved caspase- 1 activation and percentage of cleaved caspase-1-positive cells, respectively (Figures 3B, C, S5B). We did notice a slight, but non-significant, decrease in the levels of cleaved caspase- 1 in the GSDM-D KO cells when compared to wild-type Huh-7.5 cells. Lack of change in levels of cleaved caspase- 1 is to be expected as caspase- 1 is found upstream of GSDM-D in the pyroptosis pathway. Taken together, these results indicate that, in the context of $\mathrm{HCV}$ infection, there is crosstalk between pyroptotic and apoptotic pathways, with a shift from the pyroptotic to the apoptotic pathway in the event of pyroptosis inhibition. 


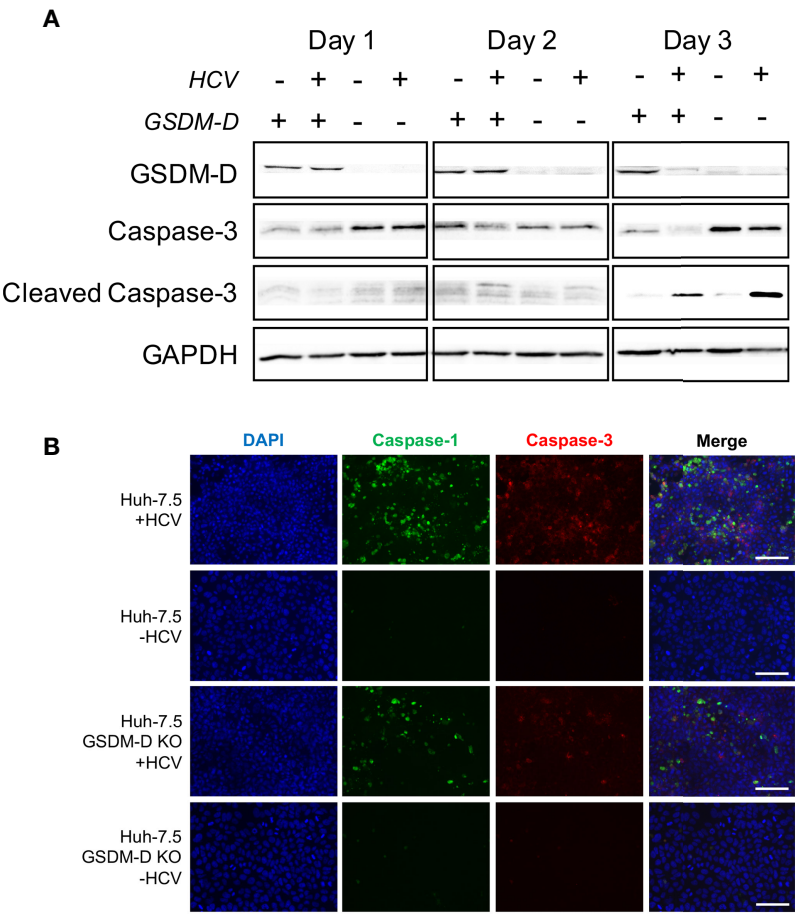

C

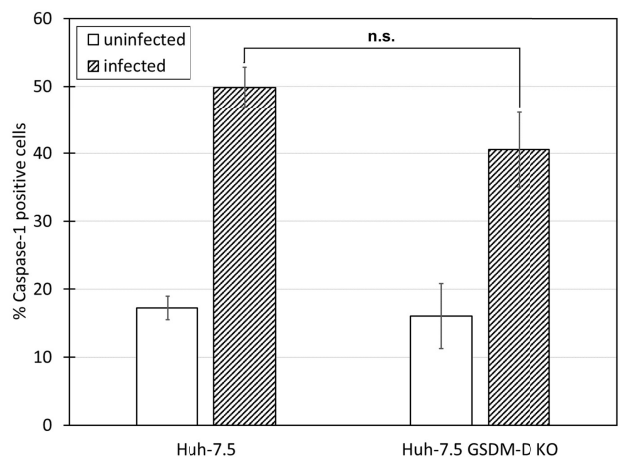

FIGURE 3 | Analysis of caspase-1 and -3 levels in the absence of GSDM-D. Huh-7.5 cells or Huh-7.5 GSDM-D KO cells were infected with HCV at MOI = 1 or left uninfected. (A) At 1, 2, and 3 days p.i., cells and culture fluids were harvested for Western Blot analysis. Membranes were probed for GSDM-D, pro-caspase-3, cleaved-caspase-3, and GAPDH. (B) At 3 days p.i., cells were stained using a specific probe for cleaved caspase-1 (green), then fixed using acetone. Cells were subsequently stained using an antibody specific for cleaved caspase-3 (red). Nuclei were stained with DAPI (blue) for observation by fluorescence microscopy. Scale bar, $100 \mu \mathrm{m}$ (C) At day 4 p.i., cells were stained for cleaved caspase-1 using a specific probe and fixed using fixative from the caspase- 1 probe kit. Cells were run on a CytoFLEX flow cytometer and data were analyzed using Kaluza analysis software. Data from GSDM-D $\mathrm{KO}$ cells are compared here to data from wild-type cells found in Figure 1D Data are presented as the percentage of total cells positive for caspase-1. $\mathrm{p}=$ n.s. (A-C) Data are representative of three independent experiments.

\section{Pyroptosis Is Reduced in the Absence of Caspase-3-Mediated Apoptosis}

More research regarding the phenomenon of crosstalk between cell death pathways has been reported in the literature in recent years $(36,37,39-43)$. We investigated potential crosstalk between the apoptotic and pyroptotic pathways and examined whether pyroptosis increased if apoptosis was inhibited in the context of HCV infection. To do this, caspase-3 KO cells were generated using a CRISPR-Cas9 approach (Figure 4A). These cells were infected with HCV or left uninfected and levels of caspase-1 and -3 were compared to wild-type Huh-7.5 cells using Western blot analysis. Surprisingly, the level of cleaved caspase- 1 in cell culture fluids was decreased in the HCV-infected KO cells compared to wild-type cells at both two and three days p.i. (Figure 4A). This reduction in the level of cleaved caspase-1 in the infected caspase-3 KO cells was also shown by fluorescence microscopy and indicates a reduction in the activation of the pyroptotic pathway (Figures 4B, S6A). Pro-caspase- 1 levels in the caspase-3 $\mathrm{KO}$ cells remained at a level consistent with that of uninfected wild-type cells (Figure 4A). Reduction of the percentage of caspase-1 positive cells in the HCV-infected caspase- $3 \mathrm{KO}$ cell population ( 25\%) compared to wild-type HCV-infected Huh7.5 cells ( $50 \%)$ was confirmed and shown to be significant using flow cytometry at day four p.i. (Figures 4C, S6B). Reduced activation of pyroptosis-associated cleaved caspase-1 in the absence of caspase-3 may indicate caspase- 3 is upstream of caspase- 1 and further supports the existence of crosstalk between the apoptotic and pyroptotic pathways.

\section{Apoptosis and Pyroptosis Are Not Mutually Exclusive Within a Single Cell}

Throughout our study, a question emerged: could the pyroptotic and apoptotic pathways be activated simultaneously or are they mutually exclusive? By performing confocal microscopy on HCV-infected Huh-7.5 cells, we were able to capture images of rare single cells that were positive for active forms of both caspase-1 and caspase-3 (Figure 5; orange-yellow colour in the merged images). This indicates that multiple cell death pathways can be activated concurrently within the same cell. The first time this was observed is documented in Figure 5A. To confirm the finding of cells positive for both caspase- 1 and -3 , we subsequently performed further microscopy experiments to obtain additional examples of this observation (Figure 5B). At this point, we cannot say whether this observation represents concurrent cell death pathways, or if we are capturing cells in transition from one cell death pathway to another.

\section{Apoptosis and Pyroptosis Liberate Infectious Virus From Cells}

Early results in this study were accompanied by a finding of decreased HCV core protein levels in the NLRP3, GSDM-D, and caspase-3 KO cells when analysis was performed via Western Blot at day three p.i. (Figure 6A). A similar phenomenon was observed in the various $\mathrm{KO}$ cells when fluorescence microscopy was performed using an antibody against $\mathrm{HCV}$ core protein at day three p.i. (Figure 6B). To determine whether the lower levels of intracellular core protein correlated with decreased infectious virus production, we performed intra- and extracellular infectious titre assays on wild-type and $\mathrm{KO}$ cells. In our virus culture system, infectious virus propagates within Huh-7.5 cells and is subsequently released into culture fluids. When using 
A

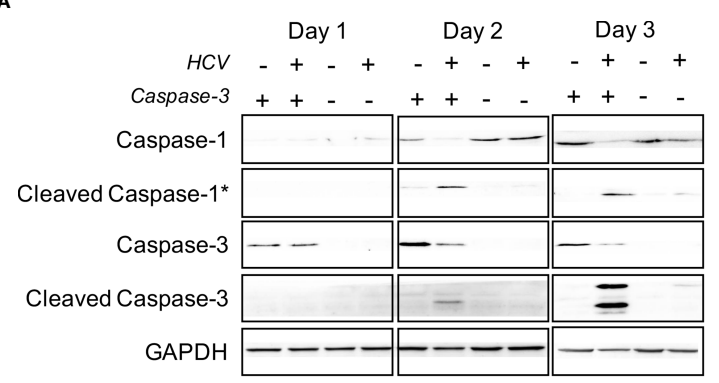

B

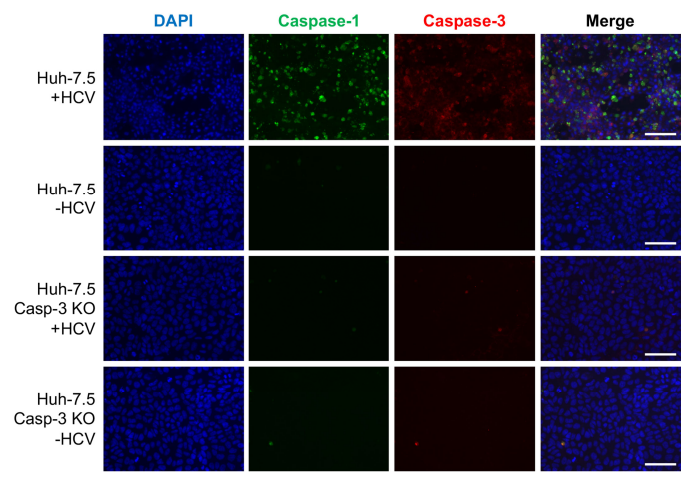

C

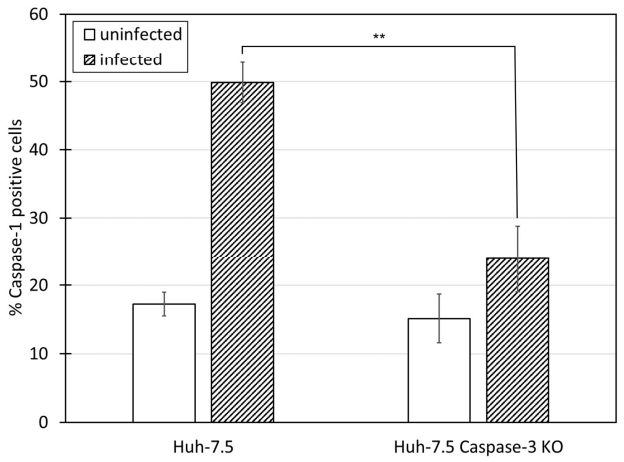

FIGURE 4 | Comparison of caspase levels when apoptosis is inhibited. Huh7.5 cells or Huh-7.5 caspase- $3 \mathrm{KO}$ cells were infected with $\mathrm{HCV}$ at $\mathrm{MOI}=1$ or left uninfected. (A) At 1, 2, and 3 days p.i., cells and culture fluids were harvested for Western Blot analysis. Membranes were probed for pro-caspase-1, cleavedcaspase-1, pro-caspase-3, cleaved-caspase-3, and GAPDH. * indicates samples from cell culture fluids rather than cell lysates. (B) At 3 days p.i., cells were stained using a probe specific for cleaved caspase-1 (green), then fixed using acetone. Cells were subsequently stained using a specific antibody for cleaved caspase-3 (red). Nuclei were stained with DAPI (blue) and analysis was by fluorescence microscopy. Scale bar, $100 \mu \mathrm{m}$ (C) At day 4 p.i., cells were stained for cleaved caspase-1 using a specific probe and fixed using fixative from the caspase- 1 probe kit. Cells were run on a CytoFLEX flow cytometer and data were analyzed using Kaluza analysis software and presented as the percent of total cells positive for caspase-1. Data from caspase- $3 \mathrm{KO}$ cells are compared here to data from wild-type cells found in Figure 1D. ${ }^{* *} p<0.005$. (A-C) Data are representative of three independent experiments.

wild-type Huh-7.5 cells, we typically see approximately 10 -fold more infectious virus in the extracellular compartment by day three p.i. compared to the intracellular compartment. When we compared intra- and extracellular titres from the $\mathrm{KO}$ cell lines to the wild-type Huh-7.5 cells, we observed a significant reduction in both intra- and extracellular titres from the $\mathrm{KO}$ cell lines (Figure 6C). An important finding to note is that the $\mathrm{KO}$ cells displayed a change in the ratio of intra- to extracellular titre, with the intracellular titre being higher, suggesting an impairment of virus release. These results indicate that both apoptosis and pyroptosis are involved in, and necessary for, efficient virus release from infected cells in vitro. While our cell culture system cannot possibly recapitulate what happens with $\mathrm{HCV}$ infection in vivo, it is possible that programmed cell death plays a role that is beneficial to the virus and contributes to the pathogenesis associated with chronic HCV infection.

\section{DISCUSSION}

Despite availability of curative drugs to treat those infected with $\mathrm{HCV}$, some individuals will still develop liver fibrosis/cirrhosis or hepatocellular carcinoma $(16,17)$. The mechanism behind the liver pathology associated with $\mathrm{HCV}$ infection, particularly following treatment with DAAs, remains a matter of significant interest. We previously proposed that pro-inflammatory programmed cell death, specifically pyroptosis, contributes to $\mathrm{HCV}$-associated liver pathogenesis (24). Our current study provides further evidence and mechanistic findings to support the potential role for virus-induced pyroptosis to promote inflammation in the liver.

The current study demonstrated that, during HCV infection, pyroptosis is initiated earlier than apoptosis. We confirmed the involvement of the NLRP3 inflammasome in $\mathrm{HCV}$-induced pyroptosis although involvement of other sensors cannot be ruled out. We also demonstrated crosstalk between the apoptosis and pyroptosis pathways within the context of HCV infection in vitro. This work also contributed to the understanding of HCV pathogenesis by demonstrating that HCV release was impaired in cell lines with various components of the pyroptotic or apoptotic pathways knocked out.

It was unsurprising that pyroptosis increased with increasing time p.i. as pyroptosis can be explained in a circular manner with the initiation of pyroptosis in one cell seeming to contribute to that of nearby cells $(1,24,44,45)$. Initiation of pyroptosis prior to apoptosis may indicate that pyroptosis is a contributing factor in the pathology associated with HCV-induced liver disease. This is particularly relevant as it is widely accepted that liver fibrosis/ cirrhosis associated with chronic infection is also associated with substantial inflammation (46). Future research should confirm this phenomenon in vivo during chronic infection. As both apoptosis and pyroptosis occur over the course of infection, these two cell death pathways likely play important and complementary roles, contributing to the progression of liver disease during chronic HCV infection.

Knockout of NLRP3 decreased caspase- 1 activation, although it was not entirely eliminated during $\mathrm{HCV}$ infection. This confirms the role of NLRP3 in HCV-induced pyroptosis but suggests it may not be the only sensor involved (Figure 7). Several sensors, including, but not limited to, RIG-I (47), IFI16 (1), NLRP9b (48), and AIM2 (49), have been shown to form inflammasomes during 
A

DAPI

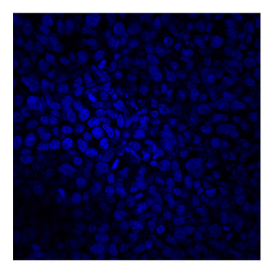

B
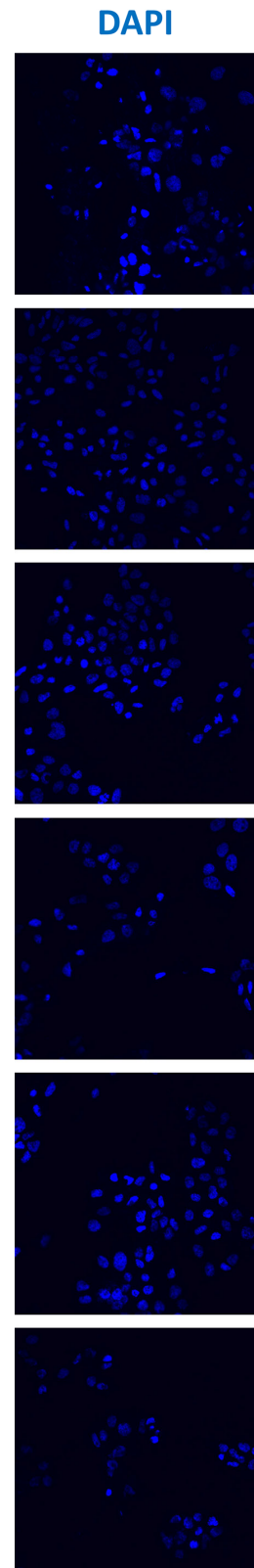

Caspase-1

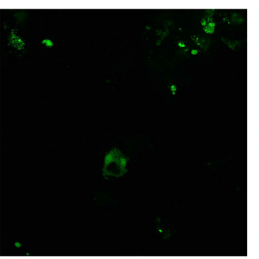

Caspase-1
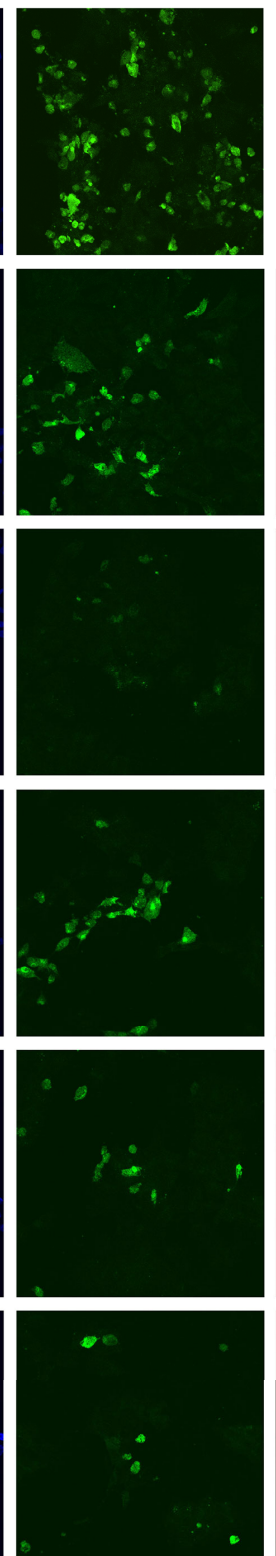
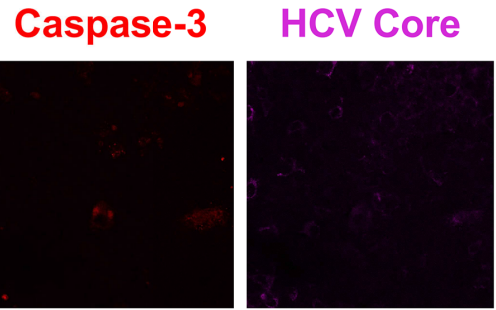

Caspase-3
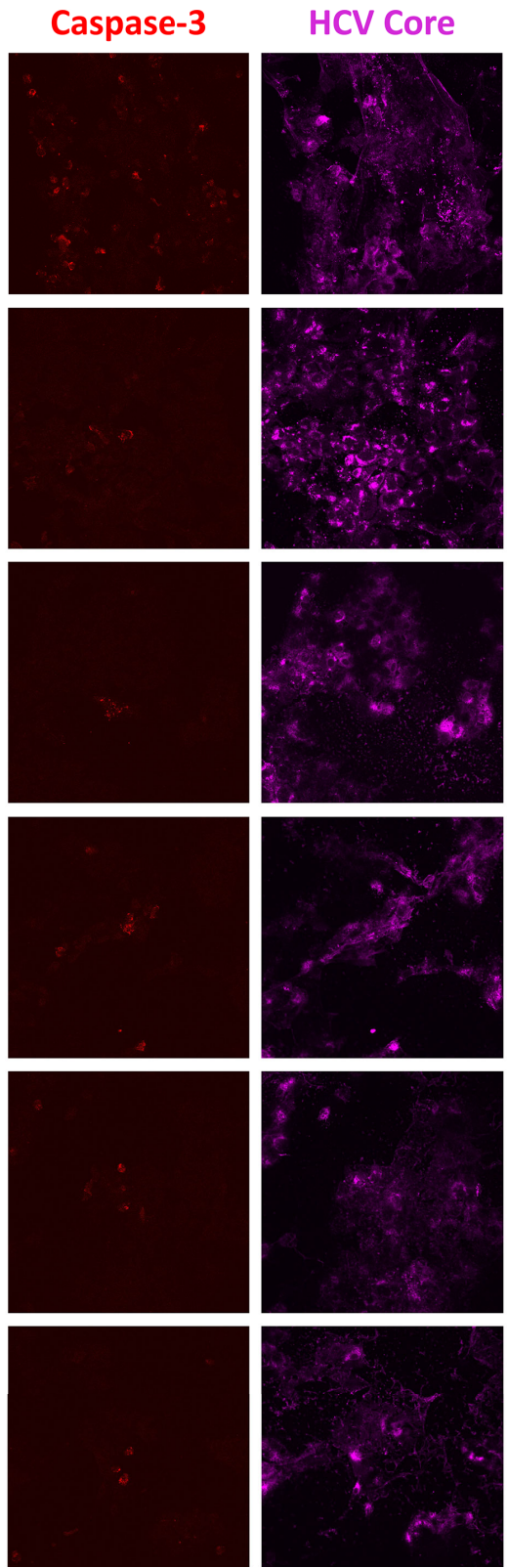

Merge

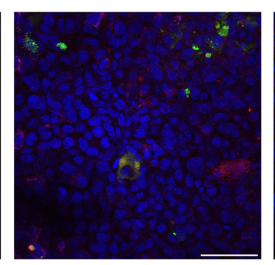

Merge
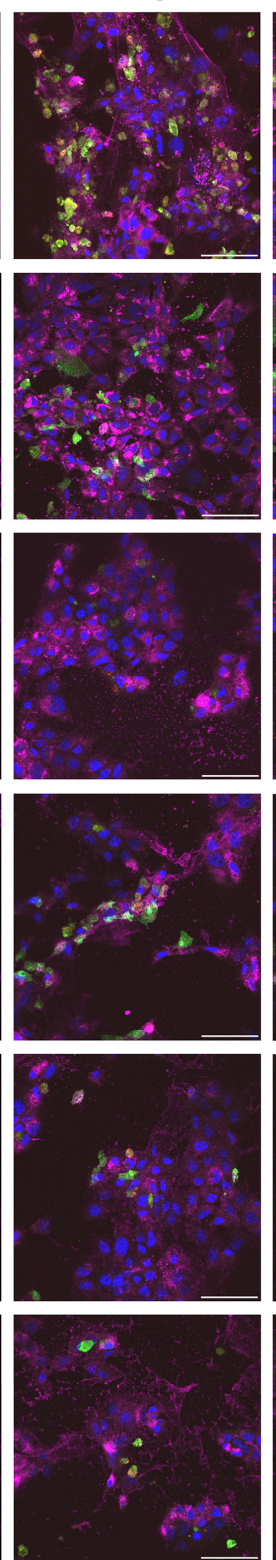

Zoom

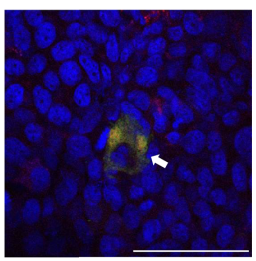

Zoom
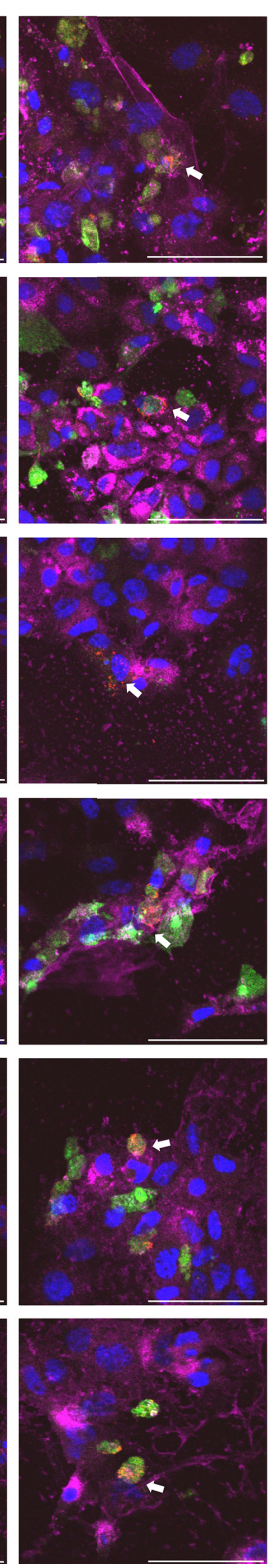

FIGURE 5 | Single cells undergoing apoptosis and pyroptosis simultaneously. Huh-7.5 cells were infected with $\mathrm{HCV}$ at MOI = 1. At 3 days p.i., cells were stained for cleaved caspase-1 (green) using a probe and then fixed using acetone. Cells were subsequently stained using antibodies specific for cleaved caspase-3 (red) and HCV core protein (magenta). Nuclei were stained with DAPI (blue) for analysis by confocal microscopy. (A) The first observed instance of a double positive cell. (B) Subsequent experiments showing additional double positive cells. (A, B) Scale bar, $100 \mu \mathrm{m}$. 
A

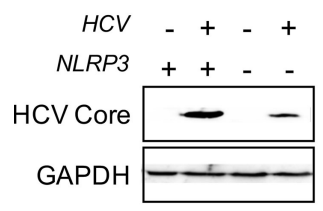

C

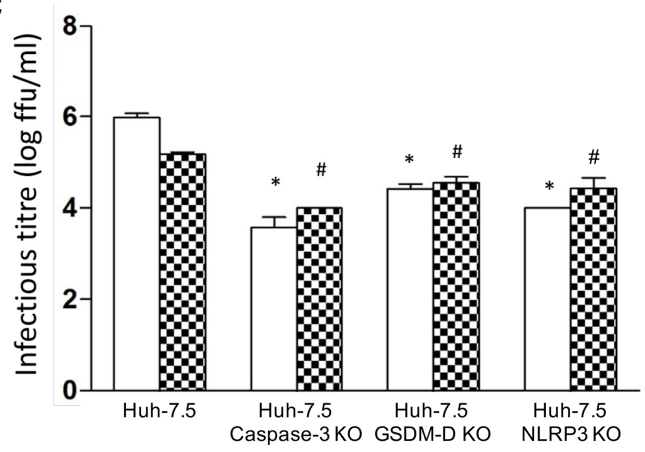

B
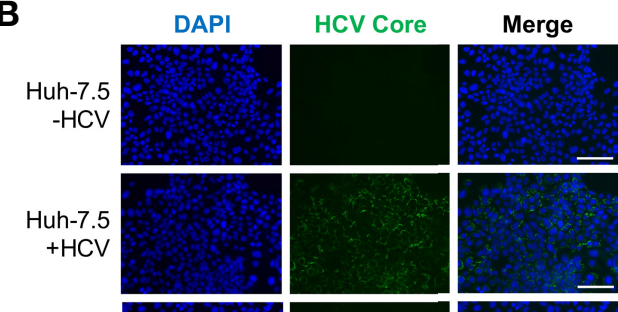

extracellular

8 intracellular
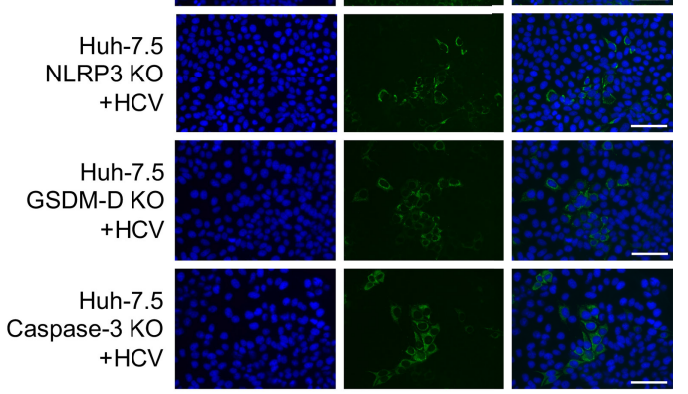

Huh-7.5

$+\mathrm{HCV}$

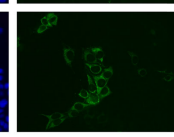

FIGURE 6 | Apoptosis and pyroptosis involvement in HCV spread. Huh-7.5 cells or Huh-7.5 KO cells (NLRP3 or GSDM-D or caspase-3) were infected with HCV at $\mathrm{MOI}=1$ or left uninfected and allowed to propagate for 3 days following infection. (A) Cells were harvested for Western Blot analysis and membranes were probed for HCV core protein and GAPDH. (B) Cells were fixed using acetone and subsequently stained using an antibody specific for HCV core protein (green) and nuclei were stained with DAPI (blue) for analysis by fluorescence microscopy. Scale bar, $100 \mu \mathrm{m}$ (C) Cells and cell culture fluids were harvested for determination of intraand extracellular titres, respectively. Titre was determined using limiting dilution assay. * indicates $p<0.05$ for differences in extracellular titre, \# indicates $p<0.05$ for differences in intracellular titre. (A-C) Data are representative of three independent experiments.

viral infection (50). It has been previously established that RNA viruses typically induce the NLRP3 inflammasome (51) while most DNA viruses induce AIM2 or IFI16 inflammasomes [reviewed in reference (50)]. However, recent research does not always support this RNA virus-NLRP3/DNA virus-AIM2 dogma (52-54). Close examination reveals that some viruses can actually activate multiple sensors, so future studies should investigate other sensors that may be triggered during HCV infection. We clearly demonstrate that HCV induces pyroptosis via the NLRP3/caspase1 pathway, but this activation of caspase-1 may also rely on other sensors.

The apoptotic and pyroptotic pathways were traditionally thought to be mutually exclusive. However, recent research has indicated there may be significantly more crosstalk than originally perceived $(36,37,39-43)$. Our findings demonstrate noteworthy crosstalk between the apoptotic and pyroptotic pathways during

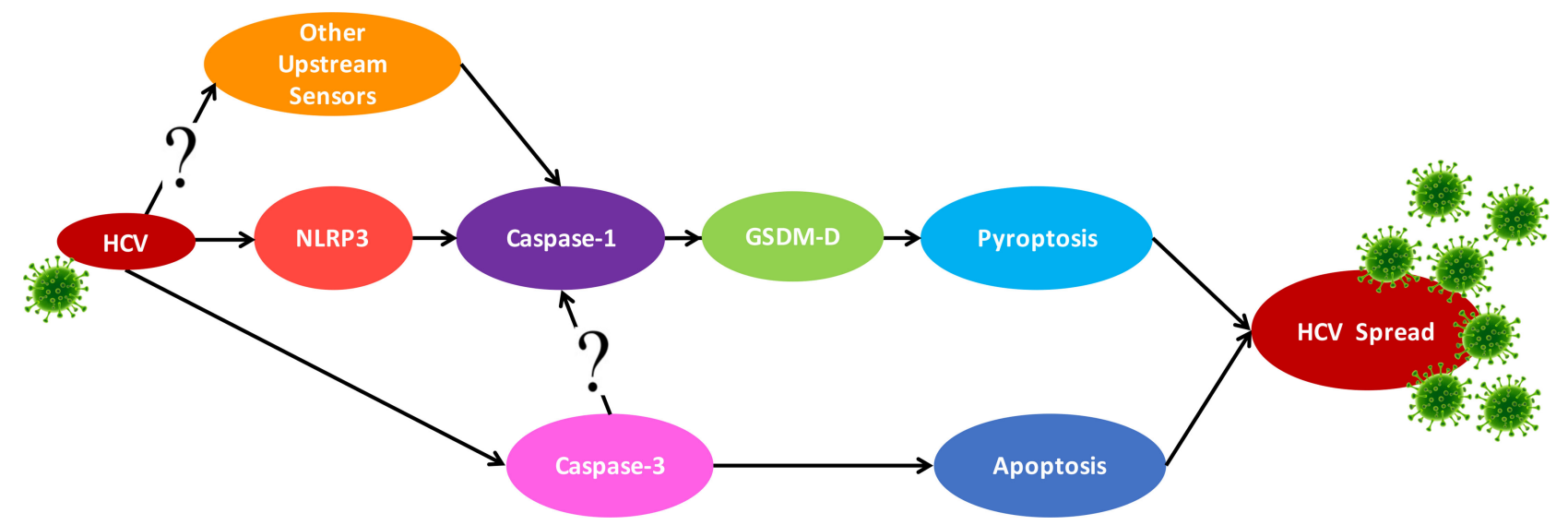

FIGURE 7 | Proposed crosstalk between the apoptosis and pyroptosis pathways. Schematic for the proposed crosstalk between the apoptosis and pyroptosis pathways and how they may contribute to HCV spread. HCV infection can trigger pyroptosis via the NLRP3 inflammasome pathway but likely also triggers inflammasome formation with other sensors. Apoptosis is also activated in the presence of HCV but this is reduced when pyroptosis is blocked. 
HCV infection (Figure 7). In the absence of GSDM-D, caspase-3 activation was increased, indicating a switch from pyroptotic cell death to apoptotic. This is in agreement with a recent study by Tsuchiya et al. (55) that demonstrates caspase- 1 initiates apoptosis in the absence of GSDM-D, which is accompanied by an increase in the expression of caspase- 3 and subsequent apoptotic cell death. This is also supported by an earlier study that used GSDM-D KO macrophages to show these cells do not die by pyroptosis, but rather by a mechanism morphologically similar to apoptosis (56). An additional study by Schneider et al. (57) showed that in the absence of GSDM-D, an alternate form of cell death was induced that utilised apoptosis-associated caspases (3 and 8) but was morphologically distinct from traditional apoptosis. As indicated in our results, we observed a change in the morphology of the cleaved caspase- 3 staining by fluorescence microscopy, rather than a clear increase as seen by Western blot. This may indicate a phenomenon more similar to the examples above, whereby, in the absence of GSDM-D, cells underwent a form of caspase-3dependent cell death morphologically distinct from apoptosis observed in wild-type cells.

In our study, caspase- $3 \mathrm{KO}$ cells showed decreased caspase-1 activation, suggesting that caspase-3 may somehow play an important role in pyroptosis induction. For example, apoptotic bodies that are not cleared by macrophages can undergo secondary pyroptosis via caspase-3-mediated cleavage of GSDM-E, resulting in cell death that is morphologically similar to pyroptosis (58). However, this does not account for the decrease in caspase-1 activation when caspase-3 was knocked out (Figure 4). It is known that GSDM-D can be cleaved by caspase-3 $(59,60)$ and it is conceivable, therefore, that in the absence of caspase-3, there would be less GSDM-D being cleaved into its active form. Here we propose that multiple sensors can trigger the pyroptosis pathway in response to HCV, that caspase3 can promote caspase- 1 activation, and that apoptosis is initiated in the absence of GSDM-D (Figure 3). Investigation into the mechanisms of crosstalk between the apoptotic and pyroptotic pathways and which proteins are involved is therefore an important area of future research.

To our knowledge, simultaneous activation of both the apoptotic and pyroptotic pathways in one cell during $\mathrm{HCV}$ infection has never before been documented (Figure 5). Significant time has been invested in optimising flow cytometry protocols to quantify what proportion of the cell population display both caspase- 1 and -3 activation, although we have yet to establish an ideal protocol.

The NLRP3, GSDM-D, and caspase-3 KO cell lines showed a decrease of both extracellular and intracellular titre upon infection compared to wild-type cells, indicating that programmed cell death is necessary for efficient HCV propagation (Figure 6). The inversion of the ratio between extracellular and intracellular titre in the $\mathrm{KO}$ cells compared to wild-type cells is important to note. This finding suggests that knocking out important cell death pathway components can affect the quantity of infectious virions able to exit the cell, with a larger proportion remaining "stuck" within the cells. Although HCV has classically been regarded as a non-lytic virus, these results suggest cell lysis may, in fact, be part of the HCV lifecycle. Taken together, these results suggest programmed cell death is an important part of efficient HCV propagation and may represent a mechanism by which $\mathrm{HCV}$ induces liver disease in vivo.

It remains generally unclear whether pyroptosis in the context of viral infection is of benefit to the host, acting as an innate antiviral response, or used as a mechanism of pathogenesis, exploited by the virus to induce disease. Classically, apoptosis has been considered an innate antiviral pathway activated by the host immune system to limit virus replication and propagation (61). However, under certain circumstances, and as identified in this study, apoptosis and pyroptosis have been found to promote both an increase of viral replication $(3,62)$ and the release of virus from infected cells, thereby potentially promoting pathogenesis $(63,64)$. It is likely that both ideas are correct in the context of different viruses. Some viruses exploit cellular pathways to induce efficient propagation and pathogenesis while others inhibit these pathways so efficient replication can occur without stimulating the innate immune responses of the cell. Here we argue that HCV utilizes the apoptotic and pyroptotic pathways of the host for efficient propagation. This is aligned with the observation that the M2 protein of influenza A virus induces pyroptosis and that efficient propagation is hindered in the absence of the M2 protein (13). Similarly, caspase-3 activation by Bovine Herpesvirus 1 results in programmed cell death accompanied by efficient virus release (65).

As mentioned, reports demonstrating crosstalk between cell death pathways have been accumulating in recent years. Some of this research has focused on PANoptosis $(36,38,66)$, the activation of pyroptosis, apoptosis, and necroptosis simultaneously within a given condition, accompanied by formation of a PANoptosome [protein complex containing RIPK1, RIPK3, NLRP3, ASC, ZDNA-binding protein 1 and caspases 1, 6, and 8; reviewed in reference (67)]. PANoptosis has been observed in other systems with various pathogens including influenza and several species of fungi $(37,38)$. At this time, we cannot confirm that PANoptosis is occurring within our $\mathrm{HCV}$ infection system without the examination of necroptosis components; however, this would be a logical and interesting follow-up of the current work.

Our study utilised a cell culture-adapted strain of $\mathrm{HCV}, \mathrm{JFH}_{\mathrm{T}}$, and human hepatoma-derived Huh-7.5 cells. While this system has been invaluable for identifying drug targets and understanding the HCV life cycle [reviewed in reference (68)], it, as with any cell culture system, does impose inherent limitations on the study. $\mathrm{JFH}_{\mathrm{T}}$ is unlike other $\mathrm{HCV}$ patient isolates in that it replicates in cell culture without adaptive mutations or modifications of the cells (69). Huh-7.5 cells are cancer-derived and are widely known to have deficiencies in pathways involved in innate immunity $(70,71)$. Due to these limitations, any findings presented here should be verified by using in vivo animal studies and biopsies from $\mathrm{HCV}$ infected patients.

Despite the in vitro limitations discussed above, the current findings should be considered within a larger context, taking into account what is already known in relation to programmed cell death in animal studies and human samples. This study cannot definitively say that these forms of programmed cell death are 
occurring in the livers of infected individuals. However, there is some literature that suggests this is the case $(22,72-75)$. In addition, there is well-documented liver inflammation in the context of HCV infection, even after treatment with DAAs (7678 ) and it is likely that pyroptotic cell death is one of the driving factors behind this inflammation. Higher levels of circulating pyroptosis-associated inflammatory cytokines (IL-18 and IL-1 $\beta$ ) have been documented in patients infected with $\operatorname{HCV}(18,79)$ but it is important to note that pyroptosis itself (i.e. inflammatory cell death) has never been documented in human liver samples directly. Moving forward, it will be crucial to identify whether pyroptosis occurs in the livers of $\mathrm{HCV}$-infected patients to identify whether the pathology we identified is physiologically/ biologically relevant.

In conclusion, we demonstrated that pyroptosis is initiated prior to apoptosis during HCV infection, with NLRP3 and possibly other sensors involved in $\mathrm{HCV}$-induced pyroptosis. There appears to be substantial crosstalk between the apoptotic and pyroptotic pathways, at least in the context of HCV infection (Figure 7). The role of this crosstalk and the mechanism by which it occurs remain to be elucidated. We show here that programmed cell death plays a role in efficient $\mathrm{HCV}$ propagation and, therefore, pathogenesis of $\mathrm{HCV}$. Since curative drugs are available to treat $\mathrm{HCV}$, future studies should address the role of programmed cell death in vivo as well as identify drug candidates whose mechanism of action is against one of these pathways in order to prevent the pathogenic effects of $\mathrm{HCV}$ that are not always mitigated with elimination of the virus $(18,76-78)$.

\section{DATA AVAILABILITY STATEMENT}

The raw data supporting the conclusions of this article will be made available by the authors upon request.

\section{AUTHOR CONTRIBUTIONS}

Conceptualization, HW, LW, KH, MG, and RR. Methodology, HW, LW, CG, CC. Investigation, HW performed fluorescence

\section{REFERENCES}

1. Doitsh G, Galloway NLK, Geng X, Yang Z, Monroe KM, Zepeda O, et al. Cell Death by Pyroptosis Drives CD4 T-Cell Depletion in HIV-1 Infection. Nature (2014) 505:509-14. doi: 10.1038/nature12940

2. Ren R, Wu S, Cai J, Yang Y, Ren X, Feng Y, et al. The H7N9 Influenza A Virus Infection Results in Lethal Inflammation in the Mammalian Host via the NLRP3-Caspase-1 Inflammasome. Sci Rep (2017) 7:7625. doi: 10.1038/ s41598-017-07384-5

3. McAuley JL, Tate MD, MacKenzie-Kludas CJ, Pinar A, Zeng W, Stutz A, et al. Activation of the NLRP3 Inflammasome by IAV Virulence Protein PB1-F2 Contributes to Severe Pathophysiology and Disease. PloS Pathog (2013) 9: e1003392. doi: 10.1371/journal.ppat.1003392

4. Bradfute SB, Swanson PE, Smith MA, Watanabe E, McDunn JE, Hotchkiss RS, et al. Mechanisms and Consequences of Ebolavirus-Induced Lymphocyte Apoptosis. J Immunol (2010) 184:327-35. doi: 10.4049/jimmunol.0901231

5. Lupfer C, Malik A, Kanneganti T-D. Inflammasome Control of Viral Infection. Curr Opin Virol (2015) 12:38-46. doi: 10.1016/j.coviro.2015.02.007 microscopy. HW and CG performed flow cytometry experiments. LW created knockout cell lines and performed Western blot analysis and titre experiments. Formal analysis, HW and CG performed the statistical analysis for flow cytometry experiments. LW performed statistical analysis for titre experiments. Writingoriginal draft, HW and LW. Writing-review and editing. HW, $\mathrm{KH}, \mathrm{MG}$, and RR. Funding acquisition, RR. Resources, KH and RR. Supervision, $\mathrm{KH}$ and RR. All authors read and revised the manuscript and gave final approval for publication.

\section{FUNDING}

HW received the Memorial University School of Graduate Studies Aldrich Award, a Memorial University Faculty of Medicine Dean's Fellowship (MSc and PhD), a Canadian Institutes of Health Research Banting and Best Canada Graduate Scholarship-Masters, and a Canadian Network on Hepatitis C Virus Doctoral Fellowship. This research was supported by research grants to RR (FRN\#PJT-159675) and KH (FRN\#PJT-153238) from the Canadian Institutes of Health Research, as well as to RR from the Medical Research Foundation, Faculty of Medicine, Memorial University. The funders had no role in study design, data collection and analysis, decision to publish, or preparation of the manuscript.

\section{ACKNOWLEDGMENTS}

We thank Memorial University's Medical Laboratories-Electron Microscopy/Flow Cytometry/Confocal Microscopy for their support of this work.

\section{SUPPLEMENTARY MATERIAL}

The Supplementary Material for this article can be found online at: https://www.frontiersin.org/articles/10.3389/fimmu.2022.788138/ full\#supplementary-material

6. Galluzzi L, Vitale I, Aaronson SA, Abrams JM, Adam D, Agostinis P, et al Molecular Mechanisms of Cell Death: Recommendations of the Nomenclature Committee on Cell Death 2018. Cell Death Differ (2018) 25:486-541. doi: 10.1038/s41418-017-0012-4

7. Marianneau P, Flamand M, Deubel V, Desprès P. Apoptotic Cell Death in Response to Dengue Virus Infection: The Pathogenesis of Dengue Haemorrhagic Fever Revisited. Clin Diagn Virol (1998) 10:113-9. doi: 10.1016/S0928-0197(98)00031-2

8. Uiprasertkul M, Kitphati R, Puthavathana P, Kriwong R, Kongchanagul A Ungchusak K, et al. Apoptosis and Pathogenesis of Avian Influenza A (H5N1) Virus in Humans. Emerg Infect Dis (2007) 13:708-12. doi: 10.3201/eid1305.060572

9. Ting JP-Y, Lovering RC, Alnemri ES, Bertin J, Boss JM, Davis BK, et al. The NLR Gene Family: A Standard Nomenclature. Immunity (2008) 28:285-7. doi: 10.1016/j.immuni.2008.02.005

10. Miao EA, Rajan JV, Aderem A. Caspase-1-Induced Pyroptotic Cell Death. Immunol Rev (2011) 243:206-14. doi: 10.1111/j.1600-065X.2011.01044.x

11. Aglietti RA, Dueber EC. Recent Insights Into the Molecular Mechanisms Underlying Pyroptosis and Gasdermin Family Functions. Trends Immunol (2017) 38:261-71. doi: 10.1016/j.it.2017.01.003 
12. Kuriakose T, Kanneganti TD. (2010). Pyroptosis in Antiviral Immunity. In: Current Topics in Microbiology and Immunology. 1-19. doi: 10.1007/ 82_2019_189

13. Ichinohe T, Pang IK, Iwasaki A. Influenza Virus Activates Inflammasomes via Its Intracellular M2 Ion Channel. Nat Immunol (2010) 11:404-10. doi: $10.1038 /$ ni.1861

14. Franchi L, Eigenbrod T, Muñoz-Planillo R, Nuñez G. The Inflammasome: A Caspase-1-Activation Platform That Regulates Immune Responses and Disease Pathogenesis. Nat Immunol (2009) 10:241-7. doi: 10.1038/ni.1703

15. WHO. Global Hepatitis Report, 2017 (2017). Available at: https://www.who. int/publications/i/item/global-hepatitis-report-2017 (Accessed August 25, 2020).

16. Ioannou GN, Green PK, Berry K. HCV Eradication Induced by Direct-Acting Antiviral Agents Reduces the Risk of Hepatocellular Carcinoma. J Hepatol (2018) 68:25-32. doi: 10.1016/j.jhep.2017.08.030

17. Kanwal F, Kramer J, Asch SM, Chayanupatkul M, Cao Y, El-Serag HB. Risk of Hepatocellular Cancer in HCV Patients Treated With Direct-Acting Antiviral Agents. Gastroenterology (2017) 153:996-1005.e1. doi: 10.1053/ j.gastro.2017.06.012

18. Burchill MA, Roby JA, Crochet N, Wind-Rotolo M, Stone AE, Edwards MG, et al. Rapid Reversal of Innate Immune Dysregulation in Blood of Patients and Livers of Humanized Mice With HCV Following DAA Therapy. PloS One (2017) 12:e0186213. doi: 10.1371/journal.pone.0186213

19. Myers RP, Krajden M, Bilodeau M, Kaita K, Marotta P, Peltekian K, et al. Burden of Disease and Cost of Chronic Hepatitis C Virus Infection in Canada. Can J Gastroenterol Hepatol (2014) 28:243-50. doi: 10.1155/2014/317623

20. Deng L, Adachi T, Kitayama K, Bungyoku Y, Kitazawa S, Ishido S, et al. Hepatitis C Virus Infection Induces Apoptosis Through a Bax-Triggered, Mitochondrion-Mediated, Caspase 3-Dependent Pathway. J Virol (2008) 82:10375-85. doi: 10.1128/JVI.00395-08

21. Zhu H, Dong H, Eksioglu E, Hemming A, Cao M, Crawford JM, et al. Hepatitis C Virus Triggers Apoptosis of a Newly Developed Hepatoma Cell Line Through Antiviral Defense System. Gastroenterology (2007) 133:164959. doi: 10.1053/j.gastro.2007.09.017

22. Bantel H, Lügering A, Poremba C, Lügering N, Held J, Domschke W, et al. Caspase Activation Correlates With the Degree of Inflammatory Liver Injury in Chronic Hepatitis C Virus Infection. Hepatology (2001) 34:758-67. doi: 10.1053/ihep.2001.28229

23. Calabrese F, Pontisso P, Pettenazzo E, Benvegnù L, Vario A, Chemello L, et al. Liver Cell Apoptosis in Chronic Hepatitis C Correlates With Histological But Not Biochemical Activity or Serum HCV-RNA Levels. Hepatology (2000) 31:1153-9. doi: 10.1053/he.2000.7123

24. Kofahi HM, Taylor NGA, Hirasawa K, Grant MD, Russell RS. Hepatitis C Virus Infection of Cultured Human Hepatoma Cells Causes Apoptosis and Pyroptosis in Both Infected and Bystander Cells. Sci Rep (2016) 6:37433. doi: $10.1038 /$ srep37433

25. Russell RS, Meunier J-C, Takikawa S, Faulk K, Engle RE, Bukh J, et al. Advantages of a Single-Cycle Production Assay to Study Cell CultureAdaptive Mutations of Hepatitis C Virus. Proc Natl Acad Sci (2008) 105:4370-5. doi: 10.1073/pnas.0800422105

26. Jones DM, Atoom AM, Zhang X, Kottilil S, Russell RS. A Genetic Interaction Between the Core and NS3 Proteins of Hepatitis C Virus Is Essential for Production of Infectious Virus. J Virol (2011) 85:12351-61. doi: 10.1128/ JVI.05313-11

27. Gaul S, Leszczynska A, Alegre F, Kaufmann B, Johnson CD, Adams LA, et al. Hepatocyte Pyroptosis and Release of Inflammasome Particles Induce Stellate Cell Activation and Liver Fibrosis. J Hepatol (2021) 74:156-67. doi: 10.1016/ j.jhep.2020.07.041

28. Baroja-Mazo A, Compan V, Martín-Sánchez F, Tapia-Abellán A, Couillin I, Pelegrín P. Early Endosome Autoantigen 1 Regulates IL-1 $\beta$ Release Upon Caspase-1 Activation Independently of Gasdermin D Membrane Permeabilization. Sci Rep (2019) 9:5788. doi: 10.1038/s41598-019-42298-4

29. Compan V, Martín-Sánchez F, Baroja-Mazo A, López-Castejón G, Gomez AI, Verkhratsky A, et al. Apoptosis-Associated Speck-Like Protein Containing a CARD Forms Specks But Does Not Activate Caspase-1 in the Absence of NLRP3 During Macrophage Swelling. J Immunol (2015) 194:1261-73. doi: 10.4049/jimmunol.1301676
30. Guo C, Fu R, Wang S, Huang Y, Li X, Zhou M, et al. NLRP3 Inflammasome Activation Contributes to the Pathogenesis of Rheumatoid Arthritis. Clin Exp Immunol (2018) 194:231-43. doi: 10.1111/cei.13167

31. Boucher D, Monteleone M, Coll RC, Chen KW, Ross CM, Teo JL, et al. Caspase-1 Self-Cleavage Is an Intrinsic Mechanism to Terminate Inflammasome Activity. J Exp Med (2018) 215:827-40. doi: 10.1084/ jem. 20172222

32. He W, Wan H, Hu L, Chen P, Wang X, Huang Z, et al. Gasdermin D Is an Executor of Pyroptosis and Required for Interleukin- $1 \beta$ Secretion. Cell Res (2015) 25:1285-98. doi: 10.1038/cr.2015.139

33. Liu X, Zhang Z, Ruan J, Pan Y, Magupalli VG, Wu H, et al. InflammasomeActivated Gasdermin D Causes Pyroptosis by Forming Membrane Pores. Nature (2016) 535:153-8. doi: 10.1038/nature18629

34. Martín-Sánchez F, Diamond C, Zeitler M, Gomez AI, Baroja-Mazo A, Bagnall $\mathrm{J}$, et al. Inflammasome-Dependent IL-1 $\beta$ Release Depends Upon Membrane Permeabilisation. Cell Death Differ (2016) 23:1219-31. doi: 10.1038/ cdd.2015.176

35. Tsuchiya K, Hosojima S, Hara H, Kushiyama H, Mahib MR, Kinoshita T, et al. Gasdermin D Mediates the Maturation and Release of IL- $1 \alpha$ Downstream of Inflammasomes. Cell Rep (2021) 34:108887. doi: 10.1016/j.celrep.2021.108887

36. Christgen S, Zheng M, Kesavardhana S, Karki R, Malireddi RKS, Banoth B, et al. Identification of the PANoptosome: A Molecular Platform Triggering Pyroptosis, Apoptosis, and Necroptosis (PANoptosis). Front Cell Infect Microbiol (2020) 10:237. doi: 10.3389/fcimb.2020.00237

37. Banoth B, Tuladhar S, Karki R, Sharma BR, Briard B, Kesavardhana S, et al. ZBP1 Promotes Fungi-Induced Inflammasome Activation and Pyroptosis, Apoptosis, and Necroptosis (PANoptosis). J Biol Chem (2020) 295:18276-83. doi: $10.1074 /$ jbc.RA120.015924

38. Kesavardhana S, Malireddi RKS, Burton AR, Porter SN, Vogel P, Pruett-Miller SM, et al. The Z $\alpha 2$ Domain of ZBP1 Is a Molecular Switch Regulating Influenza-Induced PANoptosis and Perinatal Lethality During Development. J Biol Chem (2020) 295:8325-30. doi: 10.1074/jbc.RA120.013752

39. Pierini R, Juruj C, Perret M, Jones CL, Mangeot P, Weiss DS, et al. AIM2/ASC Triggers Caspase-8-Dependent Apoptosis in Francisella-Infected Caspase-1Deficient Macrophages. Cell Death Differ (2012) 19:1709-21. doi: 10.1038/ cdd.2012.51

40. Taabazuing CY, Okondo MC, Bachovchin DA. Pyroptosis and Apoptosis Pathways Engage in Bidirectional Crosstalk in Monocytes and Macrophages. Cell Chem Biol (2017) 24:507-514.e4. doi: 10.1016/j.chembiol.2017.03.009

41. Zhivotovsky B, Orrenius S. Cell Death Mechanisms: Cross-Talk and Role in Disease. Exp Cell Res (2010) 316:1374-83. doi: 10.1016/j.yexcr.2010.02.037

42. Deng X, Zou W, Xiong M, Wang Z, Engelhardt JF, Ye SQ, et al. Human Parvovirus Infection of Human Airway Epithelia Induces Pyroptotic Cell Death by Inhibiting Apoptosis. J Virol (2017) 91:23. doi: 10.1128/JVI.01533-17

43. Lötzerich M, Roulin PS, Boucke K, Witte R, Georgiev O, Greber UF. Rhinovirus 3C Protease Suppresses Apoptosis and Triggers CaspaseIndependent Cell Death. Cell Death Dis (2018) 9:272. doi: 10.1038/s41419018-0306-6

44. Tan TY, Chu JJH. Dengue Virus-Infected Human Monocytes Trigger Late Activation of Caspase-1, Which Mediates Pro-Inflammatory IL-1 $\beta$ Secretion and Pyroptosis. J Gen Virol (2013) 94:2215-20. doi: 10.1099/vir.0.055277-0

45. Franklin BS, Bossaller L, De Nardo D, Ratter JM, Stutz A, Engels G, et al. The Adaptor ASC has Extracellular and "Prionoid" Activities That Propagate Inflammation. Nat Immunol (2014) 15:727-37. doi: 10.1038/ni.2913

46. Saile B, Ramadori G. Inflammation, Damage Repair and Liver Fibrosis - Role of Cytokines and Different Cell Types. Z Für Gastroenterol (2007) 45:77-86. doi: 10.1055/s-2006-927395

47. Poeck H, Bscheider M, Gross O, Finger K, Roth S, Rebsamen M, et al. Recognition of RNA Virus by RIG-I Results in Activation of CARD9 and Inflammasome Signaling for Interleukin $1 \beta$ Production. Nat Immunol (2010) 11:63-9. doi: 10.1038/ni.1824

48. Zhu S, Ding S, Wang P, Wei Z, Pan W, Palm NW, et al. Nlrp9b Inflammasome Restricts Rotavirus Infection in Intestinal Epithelial Cells. Nature (2017) 546:667-70. doi: 10.1038/nature22967

49. Han Y, Chen Z, Hou R, Yan D, Liu C, Chen S, et al. Expression of AIM2 is Correlated With Increased Inflammation in Chronic Hepatitis B Patients. Virol J (2015) 12:129. doi: 10.1186/s12985-015-0360-y 
50. Chen I-Y, Ichinohe T. Response of Host Inflammasomes to Viral Infection. Trends Microbiol (2015) 23:55-63. doi: 10.1016/j.tim.2014.09.007

51. Halfmann P, Hill-Batorski L, Kawaoka Y. The Induction of IL-1 $\beta$ Secretion Through the NLRP3 Inflammasome During Ebola Virus Infection. J Infect Dis (2018) 218:S504-7. doi: 10.1093/infdis/jiy433

52. de Sousa JR, Azevedo R do S da S, Martins Filho AJ, de Araujo MTF, Cruz E do RM, Vasconcelos BCB, et al. In Situ Inflammasome Activation Results in Severe Damage to the Central Nervous System in Fatal Zika Virus Microcephaly Cases. Cytokine (2018) 111:255-64. doi: 10.1016/j.cyto. 2018.08.008

53. Coulon P-G, Dhanushkodi N, Prakash S, Srivastava R, Roy S, Alomari NI, et al. NLRP3, NLRP12, and IFI16 Inflammasomes Induction and Caspase-1 Activation Triggered by Virulent HSV-1 Strains Are Associated With Severe Corneal Inflammatory Herpetic Disease. Front Immunol (2019) 10:1631. doi: 10.3389/fimmu.2019.01631

54. Yogarajah T, Ong KC, Perera D, Wong KT. AIM2 Inflammasome-Mediated Pyroptosis in Enterovirus A71-Infected Neuronal Cells Restricts Viral Replication. Sci Rep (2017) 7:5845. doi: 10.1038/s41598-017-05589-2

55. Tsuchiya K, Nakajima S, Hosojima S, Thi Nguyen D, Hattori T, Manh Le T, et al. Caspase-1 Initiates Apoptosis in the Absence of Gasdermin D. Nat Commun (2019) 10:2091. doi: 10.1038/s41467-019-09753-2

56. Kayagaki N, Stowe IB, Lee BL, O’Rourke K, Anderson K, Warming S, et al. Caspase-11 Cleaves Gasdermin D for Non-Canonical Inflammasome Signalling. Nature (2015) 526:666-71. doi: 10.1038/nature15541

57. Schneider KS, Groß CJ, Dreier RF, Saller BS, Mishra R, Gorka O, et al. The Inflammasome Drives GSDMD-Independent Secondary Pyroptosis and IL-1 Release in the Absence of Caspase-1 Protease Activity. Cell Rep (2017) 21:3846-59. doi: 10.1016/j.celrep.2017.12.018

58. Rogers C, Fernandes-Alnemri T, Mayes L, Alnemri D, Cingolani G, Alnemri ES. Cleavage of DFNA5 by Caspase-3 During Apoptosis Mediates Progression to Secondary Necrotic/Pyroptotic Cell Death. Nat Commun (2017) 8:14128. doi: $10.1038 /$ ncomms 14128

59. Orning P, Weng D, Starheim K, Ratner D, Best Z, Lee B, et al. Pathogen Blockade of TAK1 Triggers Caspase-8-Dependent Cleavage of Gasdermin D and Cell Death. Science (2018) 362:1064-9. doi: 10.1126/science.aau2818

60. Sarhan J, Liu BC, Muendlein HI, Li P, Nilson R, Tang AY, et al. Caspase-8 Induces Cleavage of Gasdermin D to Elicit Pyroptosis During Yersinia Infection. Proc Natl Acad Sci (2018) 115:E10888-97. doi: 10.1073/ pnas. 1809548115

61. Sarmento L, Li X, Howerth E, Jackson AC, Fu ZF. Glycoprotein-Mediated Induction of Apoptosis Limits the Spread of Attenuated Rabies Viruses in the Central Nervous System of Mice. J Neurovirol (2005) 11:571-81. doi: 10.1080/ 13550280500385310

62. Bauer RN, Brighton LE, Mueller L, Xiang Z, Rager JE, Fry RC, et al. Influenza Enhances Caspase-1 in Bronchial Epithelial Cells From Asthmatic Volunteers and is Associated With Pathogenesis. J Allergy Clin Immunol (2012) 130:958967.e14. doi: 10.1016/j.jaci.2012.07.013

63. Chen Y-J, Wang S-F, Weng I-C, Hong M-H, Lo T-H, Jan J-T, et al. Galectin-3 Enhances Avian H5N1 Influenza A Virus-Induced Pulmonary Inflammation by Promoting NLRP3 Inflammasome Activation. Am J Pathol (2018) 188:1031-42. doi: 10.1016/j.ajpath.2017.12.014

64. Yang Y, Xiong Z, Zhang S, Yan Y, Nguyen J, Ng B, et al. Bcl-xL Inhibits T-Cell Apoptosis Induced by Expression of SARS Coronavirus E Protein in the Absence of Growth Factors. Biochem J (2005) 392:135-43. doi: 10.1042/ BJ20050698

65. Devireddy LR, Jones CJ. Activation of Caspases and P53 by Bovine Herpesvirus 1 Infection Results in Programmed Cell Death and Efficient Virus Release. J Virol (1999) 73:3778-88. doi: 10.1128/JVI.73.5.3778-3788.1999

66. Zheng M, Williams EP, Malireddi RKS, Karki R, Banoth B, Burton A, et al. Impaired NLRP3 Inflammasome Activation/Pyroptosis Leads to Robust Inflammatory Cell Death via Caspase-8/RIPK3 During Coronavirus Infection. J Biol Chem (2020) 295:14040-52. doi: 10.1074/jbc.RA120.015036

67. Samir P, Malireddi RKS, Kanneganti T-D. The PANoptosome: A Deadly Protein Complex Driving Pyroptosis, Apoptosis, and Necroptosis (PANoptosis). Front Cell Infect Microbiol (2020) 10:238. doi: 10.3389/ fcimb.2020.00238
68. Tellinghuisen TL, Evans MJ, von Hahn T, You S, Rice CM. Studying Hepatitis C Virus: Making the Best of a Bad Virus. J Virol (2007) 81:8853-67. doi: 10.1128/JVI.00753-07

69. Kato T, Date T, Miyamoto M, Furusaka A, Tokushige K, Mizokami M, et al. Efficient Replication of the Genotype 2a Hepatitis C Virus Subgenomic Replicon. Gastroenterology (2003) 125:1808-17. doi: 10.1053/j.gastro. 2003.09.023

70. Sumpter R, Loo Y-M, Foy E, Li K, Yoneyama M, Fujita T, et al. Regulating Intracellular Antiviral Defense and Permissiveness to Hepatitis C Virus RNA Replication Through a Cellular RNA Helicase, RIG-I. J Virol (2005) 79:268999. doi: 10.1128/JVI.79.5.2689-2699.2005

71. Kawamoto M, Yamaji T, Saito K, Shirasago Y, Satomura K, Endo T, et al. Identification of Characteristic Genomic Markers in Human Hepatoma HuH7 and Huh7.5.1-8 Cell Lines. Front Genet (2020) 11:546106. doi: 10.3389/ fgene.2020.546106

72. Spanakis NE, Garinis GA, Alexopoulos EC, Patrinos GP, Menounos PG, Sklavounou A, et al. Cytokine Serum Levels in Patients With Chronic HCV Infection. J Clin Lab Anal (2002) 16:40-6. doi: 10.1002/jcla.2060

73. Chattergoon MA, Levine JS, Latanich R, Osburn WO, Thomas DL, Cox AL. High Plasma Interleukin-18 Levels Mark the Acute Phase of Hepatitis C Virus Infection. J Infect Dis (2011) 204:1730-40. doi: 10.1093/infdis/jir642

74. Valva P, Casciato P, Lezama C, Galoppo M, Gadano A, Galdame O, et al. Serum Apoptosis Markers Related to Liver Damage in Chronic Hepatitis C: Sfas as a Marker of Advanced Fibrosis in Children and Adults While M30 of Severe Steatosis Only in Children. PloS One (2013) 8:e53519. doi: 10.1371/ journal.pone.0053519

75. Debes JD, van Tilborg M, Groothuismink ZMA, Hansen BE, Schulze zur Wiesch J, von Felden J, et al. Levels of Cytokines in Serum Associate With Development of Hepatocellular Carcinoma in Patients With HCV Infection Treated With Direct-Acting Antivirals. Gastroenterology (2018) 154:515-7.e3. doi: 10.1053/j.gastro.2017.10.035

76. Welsch C, Efinger M, von Wagner M, Herrmann E, Zeuzem S, Welzel TM, et al. Ongoing Liver Inflammation in Patients With Chronic Hepatitis C and Sustained Virological Response. PloS One (2017) 12:e0171755. doi: 10.1371/ journal.pone. 0171755

77. Putra J, Schiano TD, Fiel MI. Histological Assessment of the Liver Explant in Transplanted Hepatitis C Virus Patients Achieving Sustained Virological Response With Direct-Acting Antiviral Agents. Histopathology (2018) 72:990-6. doi: 10.1111/his.13453

78. Olveira A, Domínguez L, Troya J, Arias A, Pulido F, Ryan P, et al. Persistently Altered Liver Test Results in Hepatitis C Patients After Sustained Virological Response With Direct-Acting Antivirals. J Viral Hepat (2018) 25:818-24. doi: $10.1111 /$ jvh. 12883

79. Negash AA, Ramos HJ, Crochet N, Lau DTY, Doehle B, Papic N, et al. IL-1 Production Through the NLRP3 Inflammasome by Hepatic Macrophages Links Hepatitis C Virus Infection With Liver Inflammation and Disease. PloS Pathog (2013) 9:e1003330. doi: 10.1371/journal.ppat.1003330

Conflict of Interest: The authors declare that the research was conducted in the absence of any commercial or financial relationships that could be construed as a potential conflict of interest.

Publisher's Note: All claims expressed in this article are solely those of the authors and do not necessarily represent those of their affiliated organizations, or those of the publisher, the editors and the reviewers. Any product that may be evaluated in this article, or claim that may be made by its manufacturer, is not guaranteed or endorsed by the publisher.

Copyright (๑) 2022 Wallace, Wang, Gardner, Corkum, Grant, Hirasawa and Russell. This is an open-access article distributed under the terms of the Creative Commons Attribution License (CC BY). The use, distribution or reproduction in other forums is permitted, provided the original author(s) and the copyright owner $(s)$ are credited and that the original publication in this journal is cited, in accordance with accepted academic practice. No use, distribution or reproduction is permitted which does not comply with these terms. 